\title{
Integral inequalities for $s$-convex functions via generalized conformable fractional integral operators
}

\author{
Artion Kashuri' ${ }^{1}$ Sajid lqbal' ${ }^{2}$ Rozana Liko' ${ }^{1}$, Wei Gao ${ }^{3 *}$ and Muhammad Samraiz ${ }^{4}$
}

\section{"Correspondence:}

gaowei@ynnu.edu.cn

${ }^{3}$ School of Information Science and

Technology, Yunnan Normal

University, Kunming, China

Full list of author information is

available at the end of the article

\begin{abstract}
We introduce new operators, the so-called left and right generalized conformable fractional integral operators. By using these operators we establish new Hermite-Hadamard inequalities for s-convex functions and products of two s-convex functions in the second sense. Also, we obtain two interesting identities for a differentiable function involving a generalized conformable fractional integral operator. By applying these identities we give Hermite-Hadamard and midpoint-type integral inequalities for s-convex functions. Different special cases have been identified and some known results are recovered from our general results. These results may motivate further research in different areas of pure and applied sciences.
\end{abstract}

MSC: Primary 26A51; secondary 26A33; 26D07; 26D10; 26D15

Keywords: Hermite-Hadamard inequality; Hölder inequality; Power mean inequality; Convexity; Conformable fractional integral; General fractional integral operators

\section{Introduction}

The theory of inequalities is known to play an important role in almost all areas of pure and applied sciences. Richard Bellman stated succinctly, at the Second International Conference on Mathematical Inequalities, Oberwolfach, Germany, July 30-August 5, 1978, that "there are three reasons for the study of inequalities: practical, theoretical, and aesthetic". In the last few decades the theory of inequalities has attracted the attention of great number of researchers.

We use $I$ to denote an interval in the real line $\mathbb{R}=(-\infty,+\infty)$, and $L\left[\lambda_{1}, \lambda_{2}\right]$, where $\lambda_{1}<\lambda_{2}$, the set of integrable functions on $\left[\lambda_{1}, \lambda_{2}\right]$.

Definition 1 A function $\mathfrak{h}: I \subset \mathbb{R} \longrightarrow \mathbb{R}$ is said to be convex if

$$
\mathfrak{h}\left(x \lambda_{1}+(1-x) \lambda_{2}\right) \leq x \mathfrak{h}\left(\lambda_{1}\right)+(1-x) \mathfrak{h}\left(\lambda_{2}\right)
$$

for all $\lambda_{1}, \lambda_{2} \in I$ and $x \in[0,1]$.

(c) The Author(s) 2020. This article is licensed under a Creative Commons Attribution 4.0 International License, which permits use sharing, adaptation, distribution and reproduction in any medium or format, as long as you give appropriate credit to the original author(s) and the source, provide a link to the Creative Commons licence, and indicate if changes were made. The images or other third party material in this article are included in the article's Creative Commons licence, unless indicated otherwise in a credit line to the material. If material is not included in the article's Creative Commons licence and your intended use is not permitted by statutory regulation or exceeds the permitted use, you will need to obtain permission directly from the copyright holder. To view a copy of this licence, visit http://creativecommons.org/licenses/by/4.0/. 
The interesting mean type inequality, known as the Hermite-Hadamard inequality for convex functions, is given by the following theorem.

Theorem 1 Let $\mathfrak{h}: I \subset \mathbb{R} \longrightarrow \mathbb{R}$ be a convex function, and let $\lambda_{1}, \lambda_{2} \in I$ with $\lambda_{1}<\lambda_{2}$. Then

$$
\mathfrak{h}\left(\frac{\lambda_{1}+\lambda_{2}}{2}\right) \leq \frac{1}{\lambda_{2}-\lambda_{1}} \int_{\lambda_{1}}^{\lambda_{2}} \mathfrak{h}(x) d x \leq \frac{\mathfrak{h}\left(\lambda_{1}\right)+\mathfrak{h}\left(\lambda_{2}\right)}{2} .
$$

Inequality (2) is also acknowledged as the trapezium inequality.

The trapezium inequality has an extraordinary interest due to its wide applications in the field of mathematical analysis. Authors of recent decades have studied (2) in the premises of newly invented definitions due to the motivation of convex functions. The interested readers can see the references $[2-7,9,12-15,18,19,21,22,24-28,30-32,34-37]$.

Definition 2 ([13]) A function $\mathfrak{h}:[0,+\infty) \longrightarrow \mathbb{R}$ is said to be $s$-convex $(s \in(0,1])$ in the second sense if

$$
\mathfrak{h}\left(a \lambda_{1}+(1-a) \lambda_{2}\right) \leq a^{s} \mathfrak{h}\left(\lambda_{1}\right)+(1-a)^{s} \mathfrak{h}\left(\lambda_{2}\right)
$$

for all $\lambda_{1}, \lambda_{2} \geq 0$ and $a \in[0,1]$.

The $s$-convex functions in the second sense are presented in [13]. Also, researchers started to study conformable fractional integrals; see [1, 8, 12, 18, 19, 30, 32]. Khalil et al. [17] defined the fractional integral only of order $0<\alpha \leq 1$, whereas Abdeljawad [1] generalized the definition of left and right conformable fractional integrals to any order $\alpha>0$. In 2017, Khan et al. implemented this definition by providing a class of Hermite-type inequalities.

Definition 3 Let $f:\left[\lambda_{1},+\infty\right) \longrightarrow \mathbb{R}, \xi \in(n, n+1]$, set $\zeta=\xi-n$. Then the left conformable fractional integral starting at $\lambda_{1}$ is defined by

$$
\left(I_{\xi}^{\lambda_{1}} f\right)(x)=\frac{1}{n !} \int_{\lambda_{1}}^{x}(x-\theta)^{n}\left(\theta-\lambda_{1}\right)^{\zeta-1} f(\theta) d \theta, \quad x>\lambda_{1} .
$$

Analogously, if $f:\left(-\infty, \lambda_{2}\right] \longrightarrow \mathbb{R}, \xi \in(n, n+1]$, and $\zeta=\xi-n$, then the right conformable fractional integral at $\lambda_{2}$ is defined by

$$
\left({ }^{\lambda_{2}} I_{\xi} f\right)(x)=\frac{1}{n !} \int_{x}^{\lambda_{2}}(\theta-x)^{n}\left(\lambda_{2}-\theta\right)^{\zeta-1} f(\theta) d \theta, \quad x<\lambda_{2} .
$$

Note that if $\xi=n+1$, then $\zeta=\xi-n=n+1-n=1$, where $n=0,1,2, \ldots$.

Set et al. [30] obtained a new generalized class of Hermite-Hadamard-type inequalities for $s$-convex functions by applying conformable fractional integrals.

Theorem 2 ([30]) Let $f:\left[\lambda_{1}, \lambda_{2}\right] \longrightarrow \mathbb{R}$ be a function with $0 \leq \lambda_{1}<\lambda_{2}, s \in(0,1]$, and $f \in$ $L\left[\lambda_{1}, \lambda_{2}\right]$. If $f$ is an s-convex function on $\left[\lambda_{1}, \lambda_{2}\right]$, then we have the following inequalities for 
conformable fractional integrals:

$$
\begin{aligned}
& \frac{\Gamma(\xi-n)}{\Gamma(\xi+1)} f\left(\frac{\lambda_{1}+\lambda_{2}}{2}\right) \\
& \quad \leq \frac{1}{2^{s}\left(\lambda_{2}-\lambda_{1}\right)^{\xi}}\left[\left(I_{\xi}^{\lambda_{1}} f\right)\left(\lambda_{2}\right)+\left({ }^{\lambda_{2}} I_{\xi} f\right)\left(\lambda_{1}\right)\right] \\
& \quad \leq\left[\frac{\zeta(n+s+1, \xi-n)+\zeta(n+1, \xi-n+s)}{n !}\right] \frac{\left.f\left(\lambda_{1}\right)+f\left(\lambda_{2}\right)\right]}{2^{s}}
\end{aligned}
$$

with $\xi \in(n, n+1], n=0,1,2, \ldots$, where $\Gamma$ is the Euler gamma function.

Sarikaya et al. [28] defined a function $\Phi:(0,+\infty) \longrightarrow[0,+\infty)$ satisfying the following conditions:

$$
\begin{aligned}
& \int_{0}^{1} \frac{\Phi(\theta)}{\theta} d \theta<+\infty, \\
& \frac{1}{\mathfrak{A}} \leq \frac{\Phi\left(\theta_{1}\right)}{\Phi\left(\theta_{2}\right)} \leq \mathfrak{A} \quad \text { for } \frac{1}{2} \leq \frac{\theta_{1}}{\theta_{2}} \leq 2, \\
& \frac{\Phi\left(\theta_{2}\right)}{\theta_{2}^{2}} \leq \mathfrak{B} \frac{\Phi\left(\theta_{1}\right)}{\theta_{1}^{2}} \quad \text { for } \theta_{1} \leq \theta_{2}, \\
& \left|\frac{\Phi\left(\theta_{2}\right)}{\theta_{2}^{2}}-\frac{\Phi\left(\theta_{1}\right)}{\theta_{1}^{2}}\right| \leq \mathfrak{C}\left|\theta_{2}-\theta_{1}\right| \frac{\Phi\left(\theta_{2}\right)}{\theta_{2}^{2}} \quad \text { for } \frac{1}{2} \leq \frac{\theta_{1}}{\theta_{2}} \leq 2,
\end{aligned}
$$

where $\mathfrak{A}, \mathfrak{B}, \mathfrak{C}>0$ are independent of $\theta_{2}, \theta_{1}>0$. If $\Phi\left(\theta_{2}\right) \theta_{2}^{\xi}$ is increasing for some $\xi \geq 0$ and $\frac{\Phi\left(\theta_{2}\right)}{\theta_{2}^{\zeta}}$ is decreasing for some $\zeta \geq 0$, then $\Phi$ satisfies (4)-(7); see [29]. Therefore the left-sided and right-sided generalized integral operators are defined as follows:

$$
\begin{aligned}
& \lambda_{1}^{+} I_{\Phi} f(x)=\int_{\lambda_{1}}^{x} \frac{\Phi(x-\theta)}{x-\theta} f(\theta) d \theta, \quad x>\lambda_{1}, \\
& \lambda_{2}^{-} I_{\Phi} f(x)=\int_{x}^{\lambda_{2}} \frac{\Phi(\theta-x)}{\theta-x} f(\theta) d \theta, \quad x<\lambda_{2} .
\end{aligned}
$$

The most significant element of generalized integrals is that they produce RiemannLiouville fractional integrals, $k$-Riemann-Liouville fractional integrals, Katugampola fractional integrals, and so on; see [10, 11, 16, 20, 23, 28, 29, 31, 33].

Now we are in position to introduce the following definitions of left and right generalized conformable fractional integral operators of any order $\xi>0$, where $\Phi:[0,+\infty) \longrightarrow$ $[0,+\infty)$ satisfies conditions (4) $-(7)$.

Definition 4 Let $\xi \in(n, n+1]$ and $\zeta=\xi-n$, where $n=0,1,2,3, \ldots$. The left generalized conformable fractional integral operator starting at $\lambda_{1}$ of order $\xi>0$ is defined by

$$
\lambda_{1}^{+} T_{\Phi} f(x)=\int_{\lambda_{1}}^{x} \frac{\Phi(x-\theta)}{x-\theta}\left(\theta-\lambda_{1}\right)^{\zeta-1} f(\theta) d \theta, \quad x>\lambda_{1} .
$$


Analogously, the right generalized conformable fractional integral operator of order $\xi>0$ is defined by

$$
\lambda_{2} T_{\Phi} f(x)=\int_{x}^{\lambda_{2}} \frac{\Phi(\theta-x)}{\theta-x}\left(\lambda_{2}-\theta\right)^{\zeta-1} f(\theta) d \theta, \quad x<\lambda_{2} .
$$

Remark 1 Taking $\zeta=1$ in Definition 4, we obtain the generalized fractional integral operators given from (8) and (9). Also, choosing $\Phi(t)=\frac{t^{n+1}}{n !}$ in Definition 4, we get Definition 3.

Motivated by the literature cited, our paper is organized as follows: In Sect. 2, using the new operators, we establish the so-called left and right generalized conformable fractional integral operators, new Hermite-Hadamard inequalities for $s$-convex functions and products of two $s$-convex functions in the second sense. In Sect. 3, we obtain two interesting identities for differentiable function involving generalized conformable fractional integral operator. By applying these identities we give Hermite-Hadamard and midpoint-type integral inequalities for $s$-convex functions. Various particular cases will be identified, and some known results will be recaptured from our general results. In Sect. 4, we give a brief conclusion.

\section{Hermite-Hadamard inequalities}

Throughout this study, let $\xi \in(n, n+1]$ and $\zeta=\xi-n$, where $n=0,1,2,3, \ldots$. Also, for all $\theta \in[0,1]$, we define

$$
\begin{aligned}
& \Omega_{\Phi}^{\zeta}(\theta)=\int_{0}^{\theta} \frac{\Phi\left(x\left(\lambda_{2}-\lambda_{1}\right)\right)}{x}(1-x)^{\zeta-1} d x<+\infty, \quad \lambda_{1}<\lambda_{2}, \\
& \Sigma_{\Phi, 1}^{\zeta}(x, \theta)=\int_{0}^{\theta} \frac{\Phi\left(u\left(x-\lambda_{1}\right)\right)}{u}(1-u)^{\zeta-1} d u<+\infty, \quad x>\lambda_{1}, \\
& \Sigma_{\Phi, 2}^{\zeta}(x, \theta)=\int_{0}^{\theta} \frac{\Phi\left(u\left(\lambda_{2}-x\right)\right)}{u}(1-u)^{\zeta-1} d u<+\infty, \quad x<\lambda_{2},
\end{aligned}
$$

and

$$
\Psi_{\Phi}^{\zeta}=\int_{\lambda_{1}}^{\lambda_{2}} \frac{\Phi\left(x-\lambda_{1}\right)}{x-\lambda_{1}}\left(\lambda_{2}-x\right)^{\zeta-1} d x=\int_{\lambda_{1}}^{\lambda_{2}} \frac{\Phi\left(\lambda_{2}-x\right)}{\lambda_{2}-x}\left(x-\lambda_{1}\right)^{\zeta-1} d x
$$

Let represent Hermite-Hadamard inequalities for s-convex functions in the second sense via general conformable fractional integral operators as follows.

Theorem 3 Let $f:\left[\lambda_{1}, \lambda_{2}\right] \longrightarrow \mathbb{R}$ be a positive function with $0 \leq \lambda_{1}<\lambda_{2}$ such that $f \in$ $L\left[\lambda_{1}, \lambda_{2}\right]$. Iff is s-convex in the second sense on $\left[\lambda_{1}, \lambda_{2}\right]$, then for any $\xi>0$ and $s \in(0,1]$, we have the following inequalities for generalized conformable fractional integral operators:

$$
f\left(\frac{\lambda_{1}+\lambda_{2}}{2}\right) \leq \frac{1}{2^{s} \Psi_{\Phi}^{\zeta}}\left[\lambda_{1}^{\lambda^{\prime}} T_{\Phi} f\left(\lambda_{2}\right)+\lambda_{2}^{-} T_{\Phi} f\left(\lambda_{1}\right)\right] \leq\left[\frac{f\left(\lambda_{1}\right)+f\left(\lambda_{2}\right)}{2^{s}}\right] \Delta_{\Phi}^{s, \zeta},
$$


where

$$
\Delta_{\Phi}^{s, \zeta}=\frac{1}{\left(\lambda_{2}-\lambda_{1}\right)^{s} \Psi_{\Phi}^{\zeta}} \int_{\lambda_{1}}^{\lambda_{2}} \frac{\Phi\left(x-\lambda_{1}\right)}{x-\lambda_{1}}\left(\lambda_{2}-x\right)^{\zeta-1}\left[\left(x-\lambda_{1}\right)^{s}+\left(\lambda_{2}-x\right)^{s}\right] d x
$$

and $\Psi_{\Phi}^{\zeta}$ is defined by (15).

Proof Let $x, y \in\left[\lambda_{1}, \lambda_{2}\right]$. Since $f$ is $s$-convex in the second sense on $\left[\lambda_{1}, \lambda_{2}\right]$, we have

$$
f\left(\frac{x+y}{2}\right) \leq \frac{f(x)+f(y)}{2^{s}} .
$$

Taking $x=\theta \lambda_{1}+(1-\theta) \lambda_{2}$ and $y=(1-\theta) \lambda_{1}+\theta \lambda_{2}$, we get

$$
2^{s} f\left(\frac{\lambda_{1}+\lambda_{2}}{2}\right) \leq f\left(\theta \lambda_{1}+(1-\theta) \lambda_{2}\right)+f\left((1-\theta) \lambda_{1}+\theta \lambda_{2}\right) .
$$

Multiplying both sides of inequality (18) by $\frac{\Phi\left(\theta\left(\lambda_{2}-\lambda_{1}\right)\right)}{\theta}(1-\theta)^{\zeta-1}$ and integrating the resulting inequality with respect to $\theta$ over $[0,1]$, we obtain

$$
\begin{aligned}
2^{s} f & \left(\frac{\lambda_{1}+\lambda_{2}}{2}\right) \int_{0}^{1} \frac{\Phi\left(\theta\left(\lambda_{2}-\lambda_{1}\right)\right)}{\theta}(1-\theta)^{\zeta-1} d \theta \\
\leq & \int_{0}^{1} \frac{\Phi\left(\theta\left(\lambda_{2}-\lambda_{1}\right)\right)}{\theta}(1-\theta)^{\zeta-1} f\left(\theta \lambda_{1}+(1-\theta) \lambda_{2}\right) d \theta \\
& \quad+\int_{0}^{1} \frac{\Phi\left(\theta\left(\lambda_{2}-\lambda_{1}\right)\right)}{\theta}(1-\theta)^{\zeta-1} f\left((1-\theta) \lambda_{1}+\theta \lambda_{2}\right) d \theta .
\end{aligned}
$$

So, we have

$$
2^{s} \Psi_{\Phi}^{\zeta} f\left(\frac{\lambda_{1}+\lambda_{2}}{2}\right) \leq\left[\lambda_{1}^{+} T_{\Phi} f\left(\lambda_{2}\right)+\lambda_{2}^{-} T_{\Phi} f\left(\lambda_{1}\right)\right]
$$

which means that the left side of (16) is proved. To prove the right side of (16), since $f$ is $s$-convex in the second sense on $\left[\lambda_{1}, \lambda_{2}\right]$, we have the inequalities

$$
f\left(\theta \lambda_{1}+(1-\theta) \lambda_{2}\right) \leq \theta^{s} f\left(\lambda_{1}\right)+(1-\theta)^{s} f\left(\lambda_{2}\right)
$$

and

$$
f\left((1-\theta) \lambda_{1}+\theta \lambda_{2}\right) \leq(1-\theta)^{s} f\left(\lambda_{1}\right)+\theta^{s} f\left(\lambda_{2}\right) .
$$

Adding (20) and (21), we get

$$
f\left(\theta \lambda_{1}+(1-\theta) \lambda_{2}\right)+f\left((1-\theta) \lambda_{1}+\theta \lambda_{2}\right) \leq\left[\theta^{s}+(1-\theta)^{s}\right]\left[f\left(\lambda_{1}\right)+f\left(\lambda_{2}\right)\right] .
$$

Multiplying both sides of inequality (22) by $\frac{\Phi\left(\theta\left(\lambda_{2}-\lambda_{1}\right)\right)}{\theta}(1-\theta)^{\zeta-1}$ and integrating the resulting inequality with respect to $t$ over $[0,1]$, we obtain

$$
\int_{0}^{1} \frac{\Phi\left(\theta\left(\lambda_{2}-\lambda_{1}\right)\right)}{\theta}(1-\theta)^{\zeta-1} f\left(\theta \lambda_{1}+(1-\theta) \lambda_{2}\right) d \theta
$$




$$
\begin{aligned}
& +\int_{0}^{1} \frac{\Phi\left(\theta\left(\lambda_{2}-\lambda_{1}\right)\right)}{\theta}(1-\theta)^{\zeta-1} f\left((1-\theta) \lambda_{1}+\theta \lambda_{2}\right) d \theta \\
\leq & {\left[f\left(\lambda_{1}\right)+f\left(\lambda_{2}\right)\right] \int_{0}^{1} \frac{\Phi\left(\theta\left(\lambda_{2}-\lambda_{1}\right)\right)}{\theta}(1-\theta)^{\zeta-1}\left[\theta^{s}+(1-\theta)^{s}\right] d \theta . }
\end{aligned}
$$

So, we have

$$
\left[\lambda_{1}^{+} T_{\Phi} f\left(\lambda_{2}\right)+\lambda_{2}^{-} T_{\Phi} f\left(\lambda_{1}\right)\right] \leq\left[f\left(\lambda_{1}\right)+f\left(\lambda_{2}\right)\right] \Psi_{\Phi}^{\zeta} \Delta_{\Phi}^{s, \zeta},
$$

which means that the right side of (16) is proved. The proof of Theorem 3 is completed.

Corollary 1 Taking $s=1$ in Theorem 3, we get the following inequalities for convex functions via generalized conformable fractional integral operators:

$$
f\left(\frac{\lambda_{1}+\lambda_{2}}{2}\right) \leq \frac{1}{2 \Psi_{\Phi}^{\zeta}}\left[\lambda_{1}^{+} T_{\Phi} f\left(\lambda_{2}\right)+\lambda_{2}^{-} T_{\Phi} f\left(\lambda_{1}\right)\right] \leq \frac{f\left(\lambda_{1}\right)+f\left(\lambda_{2}\right)}{2}
$$

Corollary 2 Taking $\Phi(\theta)=\frac{\theta^{n+1}}{n !}$ in Theorem 3, we get Theorem 2 .

Remark 2 Taking $\xi=s=1$ in Corollary 2, we get the well-known Hermite-Hadamard inequality (2).

Let us represent now Hermite-Hadamard inequalities for the product of two $s$-convex functions in the second sense via general conformable fractional integral operators.

Theorem 4 Let $f, g:\left[\lambda_{1}, \lambda_{2}\right] \longrightarrow \mathbb{R}$ be two positive functions with $0 \leq \lambda_{1}<\lambda_{2}$ and $f \in$ $L\left[\lambda_{1}, \lambda_{2}\right]$. If $f$ and $g$ are s-convex in the second sense on $\left[\lambda_{1}, \lambda_{2}\right]$, then for any $\xi>0$ and $s \in(0,1]$, we have the following inequalities for generalized conformable fractional integral operators:

$$
\begin{aligned}
& f\left(\frac{\lambda_{1}+\lambda_{2}}{2}\right) g\left(\frac{\lambda_{1}+\lambda_{2}}{2}\right)-\frac{1}{4^{s} \Psi_{\Phi}^{\zeta}}\left[\lambda_{1}^{+} T_{\Phi} f\left(\lambda_{2}\right) g\left(\lambda_{2}\right)+\lambda_{2}^{-} T_{\Phi} f\left(\lambda_{1}\right) g\left(\lambda_{1}\right)\right] \\
& \quad \leq \frac{1}{4^{s} \Psi_{\Phi}^{\zeta}}\left[\lambda_{1}^{+} T_{\Phi} f\left(\lambda_{2}\right) g\left(\lambda_{1}\right)+\lambda_{2}^{-} T_{\Phi} f\left(\lambda_{1}\right) g\left(\lambda_{2}\right)\right] \\
& \quad \leq \frac{1}{4^{s}}\left[2 M\left(\lambda_{1}, \lambda_{2}\right) \Theta_{\Phi}^{s, \zeta}+N\left(\lambda_{1}, \lambda_{2}\right) \Delta_{\Phi}^{2 s, \zeta}\right]
\end{aligned}
$$

where

$$
\begin{aligned}
& M\left(\lambda_{1}, \lambda_{2}\right)=f\left(\lambda_{1}\right) g\left(\lambda_{1}\right)+f\left(\lambda_{2}\right) g\left(\lambda_{2}\right), \\
& N\left(\lambda_{1}, \lambda_{2}\right)=f\left(\lambda_{1}\right) g\left(\lambda_{2}\right)+f\left(\lambda_{2}\right) g\left(\lambda_{1}\right), \\
& \Theta_{\Phi}^{s, \zeta}=\frac{1}{\left(\lambda_{2}-\lambda_{1}\right)^{2 s} \Psi_{\Phi}^{\zeta}} \int_{\lambda_{1}}^{\lambda_{2}} \frac{\Phi\left(x-\lambda_{1}\right)}{x-\lambda_{1}}\left(\lambda_{2}-x\right)^{\zeta-1}\left(x-\lambda_{1}\right)^{s}\left(\lambda_{2}-x\right)^{s} d x,
\end{aligned}
$$

and $\Psi_{\Phi}^{\zeta}$ and $\Delta_{\Phi}^{2 s, \zeta}$ are defined by (15) and (17). 
Proof Let $x, y \in\left[\lambda_{1}, \lambda_{2}\right]$. Since $f$ and $g$ are $s$-convex in the second sense on $\left[\lambda_{1}, \lambda_{2}\right]$, we have

$$
f\left(\frac{x+y}{2}\right) \leq \frac{f(x)+f(y)}{2^{s}}, \quad g\left(\frac{x+y}{2}\right) \leq \frac{g(x)+g(y)}{2^{s}} .
$$

Taking $x=\theta \lambda_{1}+(1-\theta) \lambda_{2}$ and $y=(1-\theta) \lambda_{1}+\theta \lambda_{2}$, we get

$$
2^{s} f\left(\frac{\lambda_{1}+\lambda_{2}}{2}\right) \leq f\left(\theta \lambda_{1}+(1-\theta) \lambda_{2}\right)+f\left((1-\theta) \lambda_{1}+\theta \lambda_{2}\right)
$$

and

$$
2^{s} g\left(\frac{\lambda_{1}+\lambda_{2}}{2}\right) \leq g\left(\theta \lambda_{1}+(1-\theta) \lambda_{2}\right)+g\left((1-\theta) \lambda_{1}+\theta \lambda_{2}\right) .
$$

Multiplying both sides of inequalities (29) and (30), we obtain

$$
\begin{aligned}
& 4^{s} f\left(\frac{\lambda_{1}+\lambda_{2}}{2}\right) g\left(\frac{\lambda_{1}+\lambda_{2}}{2}\right) \\
& \leq f\left(\theta \lambda_{1}+(1-\theta) \lambda_{2}\right) g\left(\theta \lambda_{1}+(1-\theta) \lambda_{2}\right) \\
&+f\left((1-\theta) \lambda_{1}+\theta \lambda_{2}\right) g\left((1-\theta) \lambda_{1}+\theta \lambda_{2}\right) \\
&+f\left(\theta \lambda_{1}+(1-\theta) \lambda_{2}\right) g\left((1-\theta) \lambda_{1}+\theta \lambda_{2}\right) \\
&+f\left((1-\theta) \lambda_{1}+\theta \lambda_{2}\right) g\left(\theta \lambda_{1}+(1-\theta) \lambda_{2}\right) .
\end{aligned}
$$

Multiplying both sides of inequality (31) by $\frac{\Phi\left(\theta\left(\lambda_{2}-\lambda_{1}\right)\right)}{\theta}(1-\theta)^{\zeta-1}$ and integrating the resulting inequality with respect to $\theta$ over $[0,1]$, we have

$$
\begin{aligned}
4^{s} f( & \left.\frac{\lambda_{1}+\lambda_{2}}{2}\right) g\left(\frac{\lambda_{1}+\lambda_{2}}{2}\right) \int_{0}^{1} \frac{\Phi\left(\theta\left(\lambda_{2}-\lambda_{1}\right)\right)}{\theta}(1-\theta)^{\zeta-1} d \theta \\
\leq & \int_{0}^{1} \frac{\Phi\left(\theta\left(\lambda_{2}-\lambda_{1}\right)\right)}{\theta}(1-\theta)^{\zeta-1} f\left(\theta \lambda_{1}+(1-\theta) \lambda_{2}\right) g\left(\theta \lambda_{1}+(1-\theta) \lambda_{2}\right) d \theta \\
& +\int_{0}^{1} \frac{\Phi\left(\theta\left(\lambda_{2}-\lambda_{1}\right)\right)}{\theta}(1-\theta)^{\zeta-1} f\left((1-\theta) \lambda_{1}+\theta \lambda_{2}\right) g\left((1-\theta) \lambda_{1}+\theta \lambda_{2}\right) d \theta \\
& +\int_{0}^{1} \frac{\Phi\left(\theta\left(\lambda_{2}-\lambda_{1}\right)\right)}{\theta}(1-\theta)^{\zeta-1} f\left(\theta \lambda_{1}+(1-\theta) \lambda_{2}\right) g\left((1-\theta) \lambda_{1}+\theta \lambda_{2}\right) d \theta \\
& +\int_{0}^{1} \frac{\Phi\left(\theta\left(\lambda_{2}-\lambda_{1}\right)\right)}{\theta}(1-\theta)^{\zeta-1} f\left((1-\theta) \lambda_{1}+\theta \lambda_{2}\right) g\left(\theta \lambda_{1}+(1-\theta) \lambda_{2}\right) d \theta .
\end{aligned}
$$

So, we get

$$
\begin{aligned}
4^{s} \Psi_{\Phi}^{\zeta} f\left(\frac{\lambda_{1}+\lambda_{2}}{2}\right) g\left(\frac{\lambda_{1}+\lambda_{2}}{2}\right) \leq & {\left[\lambda_{1}^{+} T_{\Phi} f\left(\lambda_{2}\right) g\left(\lambda_{1}\right)+\lambda_{2}^{-} T_{\Phi} f\left(\lambda_{1}\right) g\left(\lambda_{2}\right)\right] } \\
& +\left[\lambda_{1}^{+} T_{\Phi} f\left(\lambda_{2}\right) g\left(\lambda_{2}\right)+\lambda_{2}^{-} T_{\Phi} f\left(\lambda_{1}\right) g\left(\lambda_{1}\right)\right],
\end{aligned}
$$

which means that the left side of (25) is proved. To prove the right side of (25), since $f$ and $g$ are $s$-convex in the second sense on $\left[\lambda_{1}, \lambda_{2}\right]$, we have the inequalities

$$
f\left(\theta \lambda_{1}+(1-\theta) \lambda_{2}\right) \leq \theta^{s} f\left(\lambda_{1}\right)+(1-\theta)^{s} f\left(\lambda_{2}\right),
$$




$$
\begin{aligned}
& f\left((1-\theta) \lambda_{1}+\theta \lambda_{2}\right) \leq(1-\theta)^{s} f\left(\lambda_{1}\right)+\theta^{s} f\left(\lambda_{2}\right), \\
& g\left(\theta \lambda_{1}+(1-\theta) \lambda_{2}\right) \leq \theta^{s} g\left(\lambda_{1}\right)+(1-\theta)^{s} g\left(\lambda_{2}\right)
\end{aligned}
$$

and

$$
g\left((1-\theta) \lambda_{1}+\theta \lambda_{2}\right) \leq(1-\theta)^{s} g\left(\lambda_{1}\right)+\theta^{s} g\left(\lambda_{2}\right) .
$$

Applying inequalities (33) to (36), we have

$$
\begin{aligned}
& f\left(\theta \lambda_{1}+(1-\theta) \lambda_{2}\right) g\left(\theta \lambda_{1}+(1-\theta) \lambda_{2}\right)+f\left((1-\theta) \lambda_{1}+\theta \lambda_{2}\right) g\left((1-\theta) \lambda_{1}+\theta \lambda_{2}\right) \\
&+f\left(\theta \lambda_{1}+(1-\theta) \lambda_{2}\right) g\left((1-\theta) \lambda_{1}+\theta \lambda_{2}\right)+f\left((1-\theta) \lambda_{1}+\theta \lambda_{2}\right) g\left(\theta \lambda_{1}+(1-\theta) \lambda_{2}\right) \\
& \leq f\left(\theta \lambda_{1}+(1-\theta) \lambda_{2}\right) g\left(\theta \lambda_{1}+(1-\theta) \lambda_{2}\right)+f\left((1-\theta) \lambda_{1}+\theta \lambda_{2}\right) g\left((1-\theta) \lambda_{1}+\theta \lambda_{2}\right) \\
&+\left[\theta^{s} f\left(\lambda_{1}\right)+(1-\theta)^{s} f\left(\lambda_{2}\right)\right] \cdot\left[(1-\theta)^{s} g\left(\lambda_{1}\right)+\theta^{s} g\left(\lambda_{2}\right)\right] \\
&+\left[(1-\theta)^{s} f\left(\lambda_{1}\right)+\theta^{s} f\left(\lambda_{2}\right)\right] \cdot\left[\theta^{s} g\left(\lambda_{1}\right)+(1-\theta)^{s} g\left(\lambda_{2}\right)\right] \\
&= f\left(\theta \lambda_{1}+(1-\theta) \lambda_{2}\right) g\left(\theta \lambda_{1}+(1-\theta) \lambda_{2}\right)+f\left((1-\theta) \lambda_{1}+\theta \lambda_{2}\right) g\left((1-\theta) \lambda_{1}+\theta \lambda_{2}\right) \\
&+2 \theta^{s}(1-\theta)^{s} M\left(\lambda_{1}, \lambda_{2}\right)+\left[\theta^{2 s}+(1-\theta)^{2 s}\right] N\left(\lambda_{1}, \lambda_{2}\right) .
\end{aligned}
$$

Multiplying both sides of inequality (37) by $\frac{\Phi\left(\theta\left(\lambda_{2}-\lambda_{1}\right)\right)}{\theta}(1-\theta)^{\zeta-1}$ and integrating the resulting inequality with respect to $\theta$ over $[0,1]$, we obtain

$$
\begin{aligned}
\int_{0}^{1} & \frac{\Phi\left(\theta\left(\lambda_{2}-\lambda_{1}\right)\right)}{\theta}(1-\theta)^{\zeta-1} f\left(\theta \lambda_{1}+(1-\theta) \lambda_{2}\right) g\left(\theta \lambda_{1}+(1-\theta) \lambda_{2}\right) d \theta \\
& +\int_{0}^{1} \frac{\Phi\left(\theta\left(\lambda_{2}-\lambda_{1}\right)\right)}{\theta}(1-\theta)^{\zeta-1} f\left((1-\theta) \lambda_{1}+\theta \lambda_{2}\right) g\left((1-\theta) \lambda_{1}+\theta \lambda_{2}\right) d \theta \\
& +\int_{0}^{1} \frac{\Phi\left(\theta\left(\lambda_{2}-\lambda_{1}\right)\right)}{\theta}(1-\theta)^{\zeta-1} f\left(\theta \lambda_{1}+(1-\theta) \lambda_{2}\right) g\left((1-\theta) \lambda_{1}+\theta \lambda_{2}\right) d \theta \\
& +\int_{0}^{1} \frac{\Phi\left(\theta\left(\lambda_{2}-\lambda_{1}\right)\right)}{\theta}(1-\theta)^{\zeta-1} f\left((1-\theta) \lambda_{1}+\theta \lambda_{2}\right) g\left(\theta \lambda_{1}+(1-\theta) \lambda_{2}\right) d \theta \\
\leq & \int_{0}^{1} \frac{\Phi\left(\theta\left(\lambda_{2}-\lambda_{1}\right)\right)}{\theta}(1-\theta)^{\zeta-1} f\left(\theta \lambda_{1}+(1-\theta) \lambda_{2}\right) g\left(\theta \lambda_{1}+(1-\theta) \lambda_{2}\right) d \theta \\
& +\int_{0}^{1} \frac{\Phi\left(\theta\left(\lambda_{2}-\lambda_{1}\right)\right)}{\theta}(1-\theta)^{\zeta-1} f\left((1-\theta) \lambda_{1}+\theta \lambda_{2}\right) g\left((1-\theta) \lambda_{1}+\theta \lambda_{2}\right) d \theta \\
& +2 M\left(\lambda_{1}, \lambda_{2}\right) \int_{0}^{1} \frac{\Phi\left(\theta\left(\lambda_{2}-\lambda_{1}\right)\right)}{\theta}(1-\theta)^{\zeta-1} \theta^{s}(1-\theta)^{s} d \theta \\
& +N\left(\lambda_{1}, \lambda_{2}\right) \int_{0}^{1} \frac{\Phi\left(\theta\left(\lambda_{2}-\lambda_{1}\right)\right)}{\theta}(1-\theta)^{\zeta-1}\left[\theta^{2 s}+(1-\theta)^{2 s}\right] d \theta .
\end{aligned}
$$

So, we get

$$
\begin{aligned}
& {\left[\lambda_{1}^{+} T_{\Phi} f\left(\lambda_{2}\right) g\left(\lambda_{2}\right)+\lambda_{2}^{-} T_{\Phi} f\left(\lambda_{1}\right) g\left(\lambda_{1}\right)\right]+\left[\lambda_{1}^{+} T_{\Phi} f\left(\lambda_{2}\right) g\left(\lambda_{1}\right)+\lambda_{2}^{-} T_{\Phi} f\left(\lambda_{1}\right) g\left(\lambda_{2}\right)\right]} \\
& \quad \leq\left[\lambda_{1}^{+} T_{\Phi} f\left(\lambda_{2}\right) g\left(\lambda_{2}\right)+\lambda_{2}^{-} T_{\Phi} f\left(\lambda_{1}\right) g\left(\lambda_{1}\right)\right]+2 M\left(\lambda_{1}, \lambda_{2}\right) \Theta_{\Phi}^{s, \zeta}+N\left(\lambda_{1}, \lambda_{2}\right) \Psi_{\Phi}^{\zeta} \Delta_{\Phi}^{2 s, \zeta},
\end{aligned}
$$


which means that the right side of (25) is proved. The proof of Theorem 4 is completed.

Corollary 3 Taking $s=1$ in Theorem 4, we get the following inequalities for products of two convex functions via generalized conformable fractional integral operators:

$$
\begin{aligned}
& f\left(\frac{\lambda_{1}+\lambda_{2}}{2}\right) g\left(\frac{\lambda_{1}+\lambda_{2}}{2}\right)-\frac{1}{4 \Psi_{\Phi}^{\zeta}}\left[\lambda_{1}^{+} T_{\Phi} f\left(\lambda_{2}\right) g\left(\lambda_{2}\right)+\lambda_{2}^{-} T_{\Phi} f\left(\lambda_{1}\right) g\left(\lambda_{1}\right)\right] \\
& \quad \leq \frac{1}{4 \Psi_{\Phi}^{\zeta}}\left[\lambda_{1}^{+} T_{\Phi} f\left(\lambda_{2}\right) g\left(\lambda_{1}\right)+\lambda_{2}^{-} T_{\Phi} f\left(\lambda_{1}\right) g\left(\lambda_{2}\right)\right] \\
& \quad \leq \frac{1}{4}\left[2 M\left(\lambda_{1}, \lambda_{2}\right) \Theta_{\Phi}^{\zeta}+N\left(\lambda_{1}, \lambda_{2}\right) \Delta_{\Phi}^{2, \zeta}\right],
\end{aligned}
$$

where

$$
\Theta_{\Phi}^{\zeta}=\frac{1}{\left(\lambda_{2}-\lambda_{1}\right)^{2} \Psi_{\Phi}^{\zeta}} \int_{\lambda_{1}}^{\lambda_{2}} \Phi\left(x-\lambda_{1}\right)\left(\lambda_{2}-x\right)^{\zeta} d x
$$

and

$$
\Delta_{\Phi}^{2, \zeta}=\frac{1}{\left(\lambda_{2}-\lambda_{1}\right)^{2} \Psi_{\Phi}^{\zeta}} \int_{\lambda_{1}}^{\lambda_{2}} \frac{\Phi\left(x-\lambda_{1}\right)}{x-\lambda_{1}}\left(\lambda_{2}-x\right)^{\zeta-1}\left[\left(x-\lambda_{1}\right)^{2}+\left(\lambda_{2}-x\right)^{2}\right] d x
$$

Corollary 4 Taking $f=g$ in Theorem 4, we get

$$
\begin{gathered}
f^{2}\left(\frac{\lambda_{1}+\lambda_{2}}{2}\right)-\frac{1}{4^{s} \Psi_{\Phi}^{\zeta}}\left[\lambda_{1}^{+} T_{\Phi} f^{2}\left(\lambda_{2}\right)+\lambda_{2}^{-} T_{\Phi} f^{2}\left(\lambda_{1}\right)\right] \\
\quad \leq \frac{1}{4^{s} \Psi_{\Phi}^{\zeta}}\left[\lambda_{1}^{+} T_{\Phi} f\left(\lambda_{1}\right) f\left(\lambda_{2}\right)+\lambda_{2}^{-} T_{\Phi} f\left(\lambda_{1}\right) f\left(\lambda_{2}\right)\right] \\
\quad \leq \frac{1}{4^{s}}\left[2 P\left(\lambda_{1}, \lambda_{2}\right) \Theta_{\Phi}^{s, \zeta}+Q\left(\lambda_{1}, \lambda_{2}\right) \Delta_{\Phi}^{2 s, \zeta}\right],
\end{gathered}
$$

where

$$
P\left(\lambda_{1}, \lambda_{2}\right)=f^{2}\left(\lambda_{1}\right)+f^{2}\left(\lambda_{2}\right), \quad Q\left(\lambda_{1}, \lambda_{2}\right)=2 f\left(\lambda_{1}\right) f\left(\lambda_{2}\right) .
$$

\section{Some other results}

To establish the results of this section regarding general conformable fractional integral operators, we first prove the following two lemmas.

Lemma 3.1 Let $f:\left[\lambda_{1}, \lambda_{2}\right] \longrightarrow \mathbb{R}$ be a differentiable function on $\left(\lambda_{1}, \lambda_{2}\right)$. If $f^{\prime} \in L\left[\lambda_{1}, \lambda_{2}\right]$, then we have the following identity for generalized conformable fractional integrals:

$$
\begin{aligned}
& {\left[\frac{f\left(\lambda_{1}\right)+f\left(\lambda_{2}\right)}{2}\right] \Psi_{\Phi}^{\zeta}-\frac{1}{2}\left[\lambda_{1}^{+} T_{\Phi} f\left(\lambda_{2}\right)+\lambda_{2}^{-} T_{\Phi} f\left(\lambda_{1}\right)\right]} \\
& \quad=\frac{\left(\lambda_{2}-\lambda_{1}\right)^{\zeta}}{2} \int_{0}^{1}\left[\Omega_{\Phi}^{\zeta}(1-\theta)-\Omega_{\Phi}^{\zeta}(\theta)\right] f^{\prime}\left(\theta \lambda_{1}+(1-\theta) \lambda_{2}\right) d \theta,
\end{aligned}
$$

where $\Omega_{\Phi}^{\zeta}(\theta)$ is defined by (12). 
Proof We denote

$$
I_{f, \Omega_{\Phi}^{\zeta}}\left(\lambda_{1}, \lambda_{2}\right)=\frac{\left(\lambda_{2}-\lambda_{1}\right)^{\zeta}}{2} \int_{0}^{1}\left[\Omega_{\Phi}^{\zeta}(1-\theta)-\Omega_{\Phi}^{\zeta}(\theta)\right] f^{\prime}\left(\theta \lambda_{1}+(1-\theta) \lambda_{2}\right) d \theta
$$

We write (42) in the form

$$
I_{f, \Omega_{\Phi}^{\zeta}}\left(\lambda_{1}, \lambda_{2}\right)=\frac{\left(\lambda_{2}-\lambda_{1}\right)^{\zeta}}{2}\left[I_{f, \Omega_{\Phi}^{\zeta}}^{(1)}\left(\lambda_{1}, \lambda_{2}\right)-I_{f, \Omega_{\Phi}^{\zeta}}^{(2)}\left(\lambda_{1}, \lambda_{2}\right)\right]
$$

where

$$
I_{f, \Omega_{\Phi}^{\zeta}}^{(1)}\left(\lambda_{1}, \lambda_{2}\right)=\int_{0}^{1} \Omega_{\Phi}^{\zeta}(1-\theta) f^{\prime}\left(\theta \lambda_{1}+(1-\theta) \lambda_{2}\right) d \theta
$$

and

$$
I_{f, \Omega_{\Phi}^{\zeta}}^{(2)}\left(\lambda_{1}, \lambda_{2}\right)=\int_{0}^{1} \Omega_{\Phi}^{\zeta}(\theta) f^{\prime}\left(\theta \lambda_{1}+(1-\theta) \lambda_{2}\right) d \theta .
$$

Integrating by parts (44) and changing the variable of integration $x=\theta \lambda_{1}+(1-\theta) \lambda_{2}$, we get

$$
\begin{aligned}
& I_{f, \Omega_{\Phi}^{\zeta}}^{(1)}\left(\lambda_{1}, \lambda_{2}\right) \\
&=\left.\Omega_{\Phi}^{\zeta}(1-\theta) \frac{f\left(\theta \lambda_{1}+(1-\theta) \lambda_{2}\right)}{\lambda_{1}-\lambda_{2}}\right|_{0} ^{1} \\
& \quad+\frac{1}{\lambda_{1}-\lambda_{2}} \int_{0}^{1} \frac{\Phi\left((1-\theta)\left(\lambda_{2}-\lambda_{1}\right)\right)}{1-\theta}(1-(1-\theta))^{\zeta-1} f\left(\theta \lambda_{1}+(1-\theta) \lambda_{2}\right) d \theta \\
&= \Psi_{\Phi}^{\zeta} \frac{f\left(\lambda_{2}\right)}{\left(\lambda_{2}-\lambda_{1}\right)^{\zeta}}-\frac{1}{\left(\lambda_{2}-\lambda_{1}\right)^{\zeta}} \times{ }_{\lambda_{2}} T_{\Phi} f\left(\lambda_{1}\right) .
\end{aligned}
$$

Similarly, using (45), we obtain

$$
I_{f, \Omega_{\Phi}^{\zeta}}^{(2)}\left(\lambda_{1}, \lambda_{2}\right)=-\Psi_{\Phi}^{\zeta} \frac{f\left(\lambda_{1}\right)}{\left(\lambda_{2}-\lambda_{1}\right)^{\zeta}}+\frac{1}{\left(\lambda_{2}-\lambda_{1}\right)^{\zeta}} \times{ }_{\lambda_{1}^{+}} T_{\Phi} f\left(\lambda_{2}\right) .
$$

Substituting (48) and (49) into (43), we obtain the desired equality (41).

Remark 3 Taking $\Phi(\theta)=\frac{\theta^{n+1}}{n !}$ in Lemma 3.1, we get ([30], Lemma 3.1).

Lemma 3.2 Let $f:\left[\lambda_{1}, \lambda_{2}\right] \longrightarrow \mathbb{R}$ be a differentiable function on $\left(\lambda_{1}, \lambda_{2}\right)$. If $f^{\prime} \in L\left[\lambda_{1}, \lambda_{2}\right]$, then we have the following identity for generalized conformable fractional integrals:

$$
\begin{aligned}
f(x) & \Psi_{\Phi}^{\zeta}-\frac{1}{2}\left[{ }_{x^{+}} T_{\Phi} f\left(\lambda_{2}\right)+{ }_{x^{-}} T_{\Phi} f\left(\lambda_{1}\right)\right] \\
= & \frac{\left(x-\lambda_{1}\right)^{\zeta}}{2} \int_{0}^{1} \Sigma_{\Phi, 1}^{\zeta}(x, \theta) f^{\prime}\left(\theta x+(1-\theta) \lambda_{1}\right) d \theta \\
& -\frac{\left(\lambda_{2}-x\right)^{\zeta}}{2} \int_{0}^{1} \Sigma_{\Phi, 2}^{\zeta}(x, \theta) f^{\prime}\left(\theta x+(1-\theta) \lambda_{2}\right) d \theta,
\end{aligned}
$$


where $\Sigma_{\Phi, 1}^{\zeta}(x, \theta)$ and $\Sigma_{\Phi, 2}^{\zeta}(x, \theta)$ are defined by (13) and (14), respectively. We denote

$$
\begin{aligned}
& I_{f, \Sigma_{\Phi, 1}^{\zeta}, \Sigma_{\Phi, 2}^{\zeta}\left(x ; \lambda_{1}, \lambda_{2}\right)} \\
& =\frac{\left(x-\lambda_{1}\right)^{\zeta}}{2} \int_{0}^{1} \Sigma_{\Phi, 1}^{\zeta}(x, \theta) f^{\prime}\left(\theta x+(1-\theta) \lambda_{1}\right) d \theta \\
& \quad-\frac{\left(\lambda_{2}-x\right)^{\zeta}}{2} \int_{0}^{1} \Sigma_{\Phi, 2}^{\zeta}(x, \theta) f^{\prime}\left(\theta x+(1-\theta) \lambda_{2}\right) d \theta .
\end{aligned}
$$

Proof See the proof of Lemma 3.1.

Theorem 5 Let $f:\left[\lambda_{1}, \lambda_{2}\right] \longrightarrow \mathbb{R}$ be a differentiable function on $\left(\lambda_{1}, \lambda_{2}\right)$. If $f^{\prime} \in L\left[\lambda_{1}, \lambda_{2}\right]$ and $\left|f^{\prime}\right|^{q}$ is s-convex in the second sense with $s \in(0,1]$, then for $q>1$ and $\frac{1}{p}+\frac{1}{q}=1$, wehave the following inequality for generalized conformable fractional integrals:

$$
\left|I_{f, \Omega_{\Phi}^{\zeta}}\left(\lambda_{1}, \lambda_{2}\right)\right| \leq \frac{\left(\lambda_{2}-\lambda_{1}\right)^{\zeta}}{2} \sqrt[p]{\Pi_{\Phi}^{\zeta}(p)} \sqrt[q]{\frac{\left|f^{\prime}\left(\lambda_{1}\right)\right|^{q}+\left|f^{\prime}\left(\lambda_{2}\right)\right|^{q}}{s+1}},
$$

where

$$
\Pi_{\Phi}^{\zeta}(p)=2 \int_{0}^{\frac{1}{2}}\left(\int_{\theta}^{1-\theta} \frac{\Phi\left(x\left(\lambda_{2}-\lambda_{1}\right)\right)}{x}(1-x)^{\zeta-1} d x\right)^{p} d \theta
$$

Proof By Lemma 3.1, the s-convexity in the second sense of $\left|f^{\prime}\right|^{q}$, the Hölder inequality, and properties of the modulus we have

$$
\begin{aligned}
& \left|I_{f, \Omega_{\Phi}^{\zeta}}\left(\lambda_{1}, \lambda_{2}\right)\right| \\
& \quad \leq \frac{\left(\lambda_{2}-\lambda_{1}\right)^{\zeta}}{2} \int_{0}^{1}\left|\Omega_{\Phi}^{\zeta}(1-\theta)-\Omega_{\Phi}^{\zeta}(\theta)\right| f^{\prime}\left(\theta \lambda_{1}+(1-\theta) \lambda_{2}\right) \mid d \theta \\
& \quad \leq \frac{\left(\lambda_{2}-\lambda_{1}\right)^{\zeta}}{2}\left(\int_{0}^{1}\left|\Omega_{\Phi}^{\zeta}(1-\theta)-\Omega_{\Phi}^{\zeta}(\theta)\right|^{p} d \theta\right)^{\frac{1}{p}}\left(\int_{0}^{1}\left|f^{\prime}\left(\theta \lambda_{1}+(1-\theta) \lambda_{2}\right)\right|^{q} d \theta\right)^{\frac{1}{q}} \\
& \quad \leq \frac{\left(\lambda_{2}-\lambda_{1}\right)^{\zeta}}{2} \sqrt[p]{\Pi_{\Phi}^{\zeta}(p)}\left(\int_{0}^{1}\left[\theta^{s}\left|f^{\prime}\left(\lambda_{1}\right)\right|^{q}+(1-\theta)^{s}\left|f^{\prime}\left(\lambda_{2}\right)\right|^{q}\right] d \theta\right)^{\frac{1}{q}} \\
& \quad=\frac{\left(\lambda_{2}-\lambda_{1}\right)^{\zeta}}{2} \sqrt[p]{\Pi_{\Phi}^{\zeta}(p)} \sqrt[q]{\frac{\left|f^{\prime}\left(\lambda_{1}\right)\right|^{q}+\left|f^{\prime}\left(\lambda_{2}\right)\right|^{q}}{s+1}} .
\end{aligned}
$$

The proof of Theorem 5 is completed.

We point out some particular cases of Theorem 5 .

Corollary 5 Taking $\Phi(\theta)=\frac{\theta^{n+1}}{n !}$ in Theorem 5 , we get ([30], Theorem 3.2).

Corollary 6 Taking $s=1$ in Theorem 5, we have the following inequality for convex function via generalized conformable fractional integral operators:

$$
\left|I_{f, \Omega_{\Phi}^{\zeta}}\left(\lambda_{1}, \lambda_{2}\right)\right| \leq \frac{\left(\lambda_{2}-\lambda_{1}\right)^{\zeta}}{2} \sqrt[p]{\Pi_{\Phi}^{\zeta}(p)} \sqrt[q]{\frac{\left|f^{\prime}\left(\lambda_{1}\right)\right|^{q}+\left|f^{\prime}\left(\lambda_{2}\right)\right|^{q}}{2}} .
$$


Corollary 7 Taking $\left|f^{\prime}\right| \leq K$ in Theorem 5 , we obtain

$$
\left|I_{f, \Omega_{\Phi}^{\zeta}}\left(\lambda_{1}, \lambda_{2}\right)\right| \leq K \frac{\left(\lambda_{2}-\lambda_{1}\right)^{\zeta}}{2} \sqrt[q]{\frac{2}{s+1}} \sqrt[p]{\Pi_{\Phi}^{\zeta}(p)} .
$$

Theorem 6 Let $f:\left[\lambda_{1}, \lambda_{2}\right] \longrightarrow \mathbb{R}$ be a differentiable function on $\left(\lambda_{1}, \lambda_{2}\right)$. If $f^{\prime} \in L\left[\lambda_{1}, \lambda_{2}\right]$ and $\left|f^{\prime}\right|^{q}$ is s-convex in the second sense with $s \in(0,1]$, then for $q \geq 1$, we have the following inequality for generalized conformable fractional integrals:

$$
\begin{aligned}
\left|I_{f, \Omega_{\Phi}^{\zeta}}\left(\lambda_{1}, \lambda_{2}\right)\right| & \\
\leq & \frac{\left(\lambda_{2}-\lambda_{1}\right)^{\zeta}}{2 \sqrt[q]{s+1}}\left\{\left[L_{\Phi, 1}^{\zeta}\right] \sqrt[1-\frac{1}{q}]{q} \sqrt{\left(L_{\Phi, 2}^{s, \zeta}+L_{\Phi, 3}^{s, \zeta}\right)\left|f^{\prime}\left(\lambda_{1}\right)\right|^{q}+\left(\Psi_{\Phi}^{\zeta}-L_{\Phi, 4}^{s, \zeta}-L_{\Phi, 5}^{s, \zeta}\right)\left|f^{\prime}\left(\lambda_{2}\right)\right|^{q}}\right. \\
& \left.+\left[L_{\Phi, 6}^{\zeta}\right] \sqrt[1-\frac{1}{q}]{\left(\Psi_{\Phi}^{\zeta}-L_{\Phi, 7}^{s, \zeta}-L_{\Phi, 8}^{s, \zeta}\right)\left|f^{\prime}\left(\lambda_{1}\right)\right|^{q}+\left(L_{\Phi, 9}^{s, \zeta}+L_{\Phi, 10}^{s, \zeta}\right)\left|f^{\prime}\left(\lambda_{2}\right)\right|^{q}}\right\}
\end{aligned}
$$

where

$$
\begin{aligned}
& L_{\Phi, 1}^{\zeta}=\frac{1}{\left(\lambda_{2}-\lambda_{1}\right)^{\zeta}}\left[\int_{\frac{\lambda_{1}+\lambda_{2}}{2}}^{\lambda_{2}} \frac{\Phi\left(\theta-\lambda_{1}\right)}{\theta-\lambda_{1}}\left(\lambda_{2}-\theta\right)^{\zeta} d \theta+\int_{\frac{\lambda_{1}+\lambda_{2}}{2}}^{\lambda_{2}} \Phi\left(\lambda_{2}-\theta\right)\left(\theta-\lambda_{1}\right)^{\zeta-1} d \theta\right], \\
& L_{\Phi, 2}^{s, \zeta}=\frac{1}{\left(\lambda_{2}-\lambda_{1}\right)^{\zeta+s}} \int_{\frac{\lambda_{1}+\lambda_{2}}{2}}^{\lambda_{2}} \frac{\Phi\left(\theta-\lambda_{1}\right)}{\theta-\lambda_{1}}\left(\lambda_{2}-\theta\right)^{\zeta+s} d \theta \text {, } \\
& L_{\Phi, 3}^{s, \zeta}=\frac{1}{\left(\lambda_{2}-\lambda_{1}\right)^{\zeta+s}} \int_{\frac{\lambda_{1}+\lambda_{2}}{2}}^{\lambda_{2}} \Phi\left(\lambda_{2}-\theta\right)\left(\theta-\lambda_{1}\right)^{\zeta-1}\left(\lambda_{2}-\theta\right)^{s} d \theta \\
& L_{\Phi, 4}^{s, \zeta}=\frac{1}{\left(\lambda_{2}-\lambda_{1}\right)^{\zeta+s}} \int_{\frac{\lambda_{1}+\lambda_{2}}{2}}^{\lambda_{2}} \Phi\left(\theta-\lambda_{1}\right)\left(\lambda_{2}-\theta\right)^{\zeta-1}\left(\theta-\lambda_{1}\right)^{s} d \theta \\
& L_{\Phi, 5}^{s, \zeta}=\frac{1}{\left(\lambda_{2}-\lambda_{1}\right)^{\zeta+s}} \int_{\frac{\lambda_{1}+\lambda_{2}}{2}}^{\lambda_{2}} \frac{\Phi\left(\lambda_{2}-\theta\right)}{\lambda_{2}-\theta}\left(\theta-\lambda_{1}\right)^{\zeta+s} d \theta \\
& L_{\Phi, 6}^{\zeta}=\Psi_{\Phi}^{\zeta}-\frac{1}{\left(\lambda_{2}-\lambda_{1}\right)^{\zeta}}\left[\int_{\lambda_{1}}^{\frac{\lambda_{1}+\lambda_{2}}{2}} \frac{\Phi\left(\theta-\lambda_{1}\right)}{\theta-\lambda_{1}}\left(\lambda_{2}-\theta\right)^{\zeta} d \theta\right. \\
& \left.+\int_{\lambda_{1}}^{\frac{\lambda_{1}+\lambda_{2}}{2}} \Phi\left(\lambda_{2}-\theta\right)\left(\theta-\lambda_{1}\right)^{\zeta-1} d \theta\right] \\
& L_{\Phi, 7}^{s, \zeta}=\frac{1}{\left(\lambda_{2}-\lambda_{1}\right)^{\zeta+s}} \int_{\lambda_{1}}^{\frac{\lambda_{1}+\lambda_{2}}{2}} \Phi\left(\lambda_{2}-\theta\right)\left(\theta-\lambda_{1}\right)^{\zeta-1}\left(\lambda_{2}-\theta\right)^{s} d \theta \text {, } \\
& L_{\Phi, 8}^{s, \zeta}=\frac{1}{\left(\lambda_{2}-\lambda_{1}\right)^{\zeta+s}} \int_{\lambda_{1}}^{\frac{\lambda_{1}+\lambda_{2}}{2}} \frac{\Phi\left(\theta-\lambda_{1}\right)}{\theta-\lambda_{1}}\left(\lambda_{2}-\theta\right)^{\zeta+s} d \theta, \\
& L_{\Phi, 9}^{s, \zeta}=\frac{1}{\left(\lambda_{2}-\lambda_{1}\right)^{\zeta+s}} \int_{\lambda_{1}}^{\frac{\lambda_{1}+\lambda_{2}}{2}} \frac{\Phi\left(\lambda_{2}-\theta\right)}{\lambda_{2}-\theta}\left(\theta-\lambda_{1}\right)^{\zeta+s} d \theta, \\
& L_{\Phi, 10}^{s, \zeta}=\frac{1}{\left(\lambda_{2}-\lambda_{1}\right)^{\zeta+s}} \int_{\lambda_{1}}^{\frac{\lambda_{1}+\lambda_{2}}{2}} \Phi\left(\theta-\lambda_{1}\right)\left(\lambda_{2}-\theta\right)^{\zeta-1}\left(\theta-\lambda_{1}\right)^{s} d \theta,
\end{aligned}
$$

and $\Psi_{\Phi}^{\zeta}$ is defined by (15). 
Proof By Lemma 3.1, the $s$-convexity in the second sense of $\left|f^{\prime}\right|^{q}$, the well-known power mean inequality, and properties of the modulus we have

$$
\begin{aligned}
& \left|I_{f, \Omega_{\Phi}^{\zeta}}\left(\lambda_{1}, \lambda_{2}\right)\right| \\
& \leq \frac{\left(\lambda_{2}-\lambda_{1}\right)^{\zeta}}{2} \int_{0}^{1}\left|\Omega_{\Phi}^{\zeta}(1-\theta)-\Omega_{\Phi}^{\zeta}(\theta)\right|\left|f^{\prime}\left(\theta \lambda_{1}+(1-\theta) \lambda_{2}\right)\right| d \theta \\
& \leq \frac{\left(\lambda_{2}-\lambda_{1}\right)^{\zeta}}{2}\left(\int_{0}^{1}\left|\Omega_{\Phi}^{\zeta}(1-\theta)-\Omega_{\Phi}^{\zeta}(\theta)\right| d \theta\right)^{1-\frac{1}{q}} \\
& \times\left(\int_{0}^{1}\left|\Omega_{\Phi}^{\zeta}(1-\theta)-\Omega_{\Phi}^{\zeta}(\theta)\right|\left|f^{\prime}\left(\theta \lambda_{1}+(1-\theta) \lambda_{2}\right)\right|^{q} d \theta\right)^{\frac{1}{q}} \\
& \leq \frac{\left(\lambda_{2}-\lambda_{1}\right)^{\zeta}}{2}\left\{\left(\int_{0}^{\frac{1}{2}}\left[\Omega_{\Phi}^{\zeta}(1-\theta)-\Omega_{\Phi}^{\zeta}(\theta)\right] d \theta\right)^{1-\frac{1}{q}}\right. \\
& \times\left(\int_{0}^{\frac{1}{2}}\left[\Omega_{\Phi}^{\zeta}(1-\theta)-\Omega_{\Phi}^{\zeta}(\theta)\right]\left[\theta^{s}\left|f^{\prime}\left(\lambda_{1}\right)\right|^{q}+(1-\theta)^{s}\left|f^{\prime}\left(\lambda_{2}\right)\right|^{q}\right] d \theta\right)^{\frac{1}{q}} \\
& +\left(\int_{\frac{1}{2}}^{1}\left[\Omega_{\Phi}^{\zeta}(\theta)-\Omega_{\Phi}^{\zeta}(1-\theta)\right] d \theta\right)^{1-\frac{1}{q}} \\
& \left.\times\left(\int_{\frac{1}{2}}^{1}\left[\Omega_{\Phi}^{\zeta}(\theta)-\Omega_{\Phi}^{\zeta}(1-\theta)\right]\left[\theta^{s}\left|f^{\prime}\left(\lambda_{1}\right)\right|^{q}+(1-\theta)^{s}\left|f^{\prime}\left(\lambda_{2}\right)\right|^{q}\right] d \theta\right)^{\frac{1}{q}}\right\} \\
& =\frac{\left(\lambda_{2}-\lambda_{1}\right)^{\zeta}}{2 \sqrt[q]{s+1}}\left\{\left[L_{\Phi, 1}^{\zeta}\right] \sqrt[1-\frac{1}{q}]{q} \sqrt{\left(L_{\Phi, 2}^{s, \zeta}+L_{\Phi, 3}^{s, \zeta}\right)\left|f^{\prime}\left(\lambda_{1}\right)\right|^{q}+\left(\Psi_{\Phi}^{\zeta}-L_{\Phi, 4}^{s, \zeta}-L_{\Phi, 5}^{s, \zeta}\right)\left|f^{\prime}\left(\lambda_{2}\right)\right|^{q}}\right. \\
& \left.+\left[L_{\Phi, 6}^{\zeta}\right] \sqrt[1-\frac{1}{q}]{q} \sqrt{\left(\Psi_{\Phi}^{\zeta}-L_{\Phi, 7}^{s, \zeta}-L_{\Phi, 8}^{s, \zeta}\right)\left|f^{\prime}\left(\lambda_{1}\right)\right|^{q}+\left(L_{\Phi, 9}^{s, \zeta}+L_{\Phi, 10}^{s, \zeta}\right)\left|f^{\prime}\left(\lambda_{2}\right)\right|^{q}}\right\} .
\end{aligned}
$$

The proof of Theorem 6 is completed.

We point out some particular cases of Theorem 6 .

Corollary 8 Taking $\Phi(\theta)=\frac{\theta^{n+1}}{n !}$ and $q=1$ in Theorem 6, we get ([30], Theorem 3.1).

Corollary 9 Taking $s=1$ in Theorem 6, we get the following inequality for convex function via generalized conformable fractional integral operators:

$$
\begin{aligned}
& \left|I_{f, \Omega_{\Phi}^{\zeta}}\left(\lambda_{1}, \lambda_{2}\right)\right| \\
& \leq \leq \frac{\left(\lambda_{2}-\lambda_{1}\right)^{\zeta}}{2 \sqrt[q]{2}}\left\{\left[L_{\Phi, 1}^{\zeta}\right] \sqrt{1-\frac{1}{q}} \sqrt[q]{\left(L_{\Phi, 2}^{\zeta}+L_{\Phi, 3}^{\zeta}\right)\left|f^{\prime}\left(\lambda_{1}\right)\right|^{q}+\left(\Psi_{\Phi}^{\zeta}-L_{\Phi, 4}^{\zeta}-L_{\Phi, 5}^{\zeta}\right)\left|f^{\prime}\left(\lambda_{2}\right)\right|^{q}}\right. \\
& \left.\quad+\left[L_{\Phi, 6}^{\zeta}\right] \sqrt[1-\frac{1}{q}]{\left(\Psi_{\Phi}^{\zeta}-L_{\Phi, 7}^{\zeta}-L_{\Phi, 8}^{\zeta}\right)\left|f^{\prime}\left(\lambda_{1}\right)\right|^{q}+\left(L_{\Phi, 9}^{\zeta}+L_{\Phi, 10}^{\zeta}\right)\left|f^{\prime}\left(\lambda_{2}\right)\right|^{q}}\right\}
\end{aligned}
$$

where

$$
\begin{aligned}
L_{\Phi, 2}^{\zeta} & =\frac{1}{\left(\lambda_{2}-\lambda_{1}\right)^{\zeta+1}} \int_{\frac{\lambda_{1}+\lambda_{2}}{2}}^{\lambda_{2}} \frac{\Phi\left(\theta-\lambda_{1}\right)}{\theta-\lambda_{1}}\left(\lambda_{2}-\theta\right)^{\zeta+1} d \theta, \\
L_{\Phi, 3}^{\zeta} & =\frac{1}{\left(\lambda_{2}-\lambda_{1}\right)^{\zeta+1}} \int_{\frac{\lambda_{1}+\lambda_{2}}{2}}^{\lambda_{2}} \Phi\left(\lambda_{2}-\theta\right)\left(\theta-\lambda_{1}\right)^{\zeta-1}\left(\lambda_{2}-\theta\right) d \theta,
\end{aligned}
$$




$$
\begin{aligned}
L_{\Phi, 4}^{\zeta} & =\frac{1}{\left(\lambda_{2}-\lambda_{1}\right)^{\zeta+1}} \int_{\frac{\lambda_{1}+\lambda_{2}}{2}}^{\lambda_{2}} \Phi\left(\theta-\lambda_{1}\right)\left(\lambda_{2}-\theta\right)^{\zeta-1}\left(\theta-\lambda_{1}\right) d \theta, \\
L_{\Phi, 5}^{\zeta} & =\frac{1}{\left(\lambda_{2}-\lambda_{1}\right)^{\zeta+1}} \int_{\frac{\lambda_{1}+\lambda_{2}}{2}}^{\lambda_{2}} \frac{\Phi\left(\lambda_{2}-\theta\right)}{\lambda_{2}-\theta}\left(\theta-\lambda_{1}\right)^{\zeta+1} d \theta, \\
L_{\Phi, 7}^{\zeta} & =\frac{1}{\left(\lambda_{2}-\lambda_{1}\right)^{\zeta+1}} \int_{\lambda_{1}}^{\frac{\lambda_{1}+\lambda_{2}}{2}} \Phi\left(\lambda_{2}-\theta\right)\left(\theta-\lambda_{1}\right)^{\zeta-1}\left(\lambda_{2}-\theta\right) d \theta, \\
L_{\Phi, 8}^{\zeta} & =\frac{1}{\left(\lambda_{2}-\lambda_{1}\right)^{\zeta+1}} \int_{\lambda_{1}}^{\frac{\lambda_{1}+\lambda_{2}}{2}} \frac{\Phi\left(\theta-\lambda_{1}\right)}{\theta-\lambda_{1}}\left(\lambda_{2}-\theta\right)^{\zeta+1} d \theta, \\
L_{\Phi, 9}^{\zeta} & =\frac{1}{\left(\lambda_{2}-\lambda_{1}\right)^{\zeta+1}} \int_{\lambda_{1}}^{\frac{\lambda_{1}+\lambda_{2}}{2}} \frac{\Phi\left(\lambda_{2}-\theta\right)}{\lambda_{2}-\theta}\left(\theta-\lambda_{1}\right)^{\zeta+1} d \theta,
\end{aligned}
$$

and

$$
L_{\Phi, 10}^{\zeta}=\frac{1}{\left(\lambda_{2}-\lambda_{1}\right)^{\zeta+1}} \int_{\lambda_{1}}^{\frac{\lambda_{1}+\lambda_{2}}{2}} \Phi\left(\theta-\lambda_{1}\right)\left(\lambda_{2}-\theta\right)^{\zeta-1}\left(\theta-\lambda_{1}\right) d \theta
$$

Corollary 10 Taking $\left|f^{\prime}\right| \leq K$ in Theorem 6 , we obtain

$$
\begin{aligned}
\left|I_{f, \Omega_{\Phi}^{\zeta}}\left(\lambda_{1}, \lambda_{2}\right)\right| & \\
\leq & K \frac{\left(\lambda_{2}-\lambda_{1}\right)^{\zeta}}{2 \sqrt[q]{s+1}}\left\{\left[L_{\Phi, 1}^{\zeta}\right] \sqrt[1-\frac{1}{q}]{q} \sqrt{\left(L_{\Phi, 2}^{s, \zeta}+L_{\Phi, 3}^{s, \zeta}\right)+\left(\Psi_{\Phi}^{\zeta}-L_{\Phi, 4}^{s, \zeta}-L_{\Phi, 5}^{s, \zeta}\right)}\right. \\
& \left.+\left[L_{\Phi, 6}^{\zeta}\right] \sqrt[1-\frac{1}{q}]{q} \sqrt{\left(\Psi_{\Phi}^{\zeta}-L_{\Phi, 7}^{s, \zeta}-L_{\Phi, 8}^{s, \zeta}\right)+\left(L_{\Phi, 9}^{s, \zeta}+L_{\Phi, 10}^{s, \zeta}\right)}\right\} .
\end{aligned}
$$

Theorem 7 Let $f:\left[\lambda_{1}, \lambda_{2}\right] \longrightarrow \mathbb{R}$ be a differentiable function on $\left(\lambda_{1}, \lambda_{2}\right)$. If $f^{\prime} \in L\left[\lambda_{1}, \lambda_{2}\right]$ and $\left|f^{\prime}\right|^{q}$ is s-convex in the second sense with $s \in(0,1]$, then for $q>1$ and $\frac{1}{p}+\frac{1}{q}=1$, we have the following inequality for generalized conformable fractional integrals:

$$
\begin{aligned}
& \mid I_{f, \Sigma_{\Phi, 1}^{\zeta}, \Sigma_{\Phi, 2}^{\zeta}\left(x ; \lambda_{1}, \lambda_{2}\right) \mid} \\
& \leq \frac{\left(x-\lambda_{1}\right)^{\zeta}}{2} \sqrt[p]{\Xi_{\Phi, 1}^{\zeta}(x, p)} \sqrt[q]{\frac{\left|f^{\prime}(x)\right|^{q}+\left|f^{\prime}\left(\lambda_{1}\right)\right|^{q}}{s+1}} \\
& \quad+\frac{\left(\lambda_{2}-x\right)^{\zeta}}{2} \sqrt[p]{\Xi_{\Phi, 2}^{\zeta}(x, p)} \sqrt[q]{\frac{\left|f^{\prime}(x)\right|^{q}+\left|f^{\prime}\left(\lambda_{2}\right)\right|^{q}}{s+1}}
\end{aligned}
$$

where

$$
\Xi_{\Phi, 1}^{\zeta}(x, p)=\int_{0}^{1}\left[\Sigma_{\Phi, 1}^{\zeta}(x, \theta)\right]^{p} d \theta, \quad \Xi_{\Phi, 2}^{\zeta}(x, p)=\int_{0}^{1}\left[\Sigma_{\Phi, 2}^{\zeta}(x, \theta)\right]^{p} d \theta
$$

Proof By Lemma 3.2, the s-convexity in the second sense of $\left|f^{\prime}\right|^{q}$, the Hölder inequality, and properties of the modulus we have

$$
\begin{aligned}
& \mid I_{f, \Sigma_{\phi, 1}^{\zeta}, \Sigma_{\Phi, 2}^{\zeta}\left(x ; \lambda_{1}, \lambda_{2}\right) \mid} \\
& \quad \leq \frac{\left(x-\lambda_{1}\right)^{\zeta}}{2} \int_{0}^{1} \Sigma_{\Phi, 1}^{\zeta}(x, \theta)\left|f^{\prime}\left(\theta x+(1-\theta) \lambda_{1}\right)\right| d \theta
\end{aligned}
$$




$$
\begin{aligned}
& +\frac{\left(\lambda_{2}-x\right)^{\zeta}}{2} \int_{0}^{1} \Sigma_{\Phi, 2}^{\zeta}(x, \theta)\left|f^{\prime}\left(\theta x+(1-\theta) \lambda_{2}\right)\right| d \theta \\
\leq & \frac{\left(x-\lambda_{1}\right)^{\zeta}}{2}\left(\int_{0}^{1}\left[\Sigma_{\Phi, 1}^{\zeta}(x, \theta)\right]^{p} d \theta\right)^{\frac{1}{p}}\left(\int_{0}^{1}\left|f^{\prime}\left(\theta x+(1-\theta) \lambda_{1}\right)\right|^{q} d \theta\right)^{\frac{1}{q}} \\
& +\frac{\left(\lambda_{2}-x\right)^{\zeta}}{2}\left(\int_{0}^{1}\left[\Sigma_{\Phi, 2}^{\zeta}(x, \theta)\right]^{p} d \theta\right)^{\frac{1}{p}}\left(\int_{0}^{1}\left|f^{\prime}\left(\theta x+(1-\theta) \lambda_{2}\right)\right|^{q} d \theta\right)^{\frac{1}{q}} \\
\leq & \frac{\left(x-\lambda_{1}\right)^{\zeta}}{2} \sqrt[p]{\Xi_{\Phi, 1}^{\zeta}(x, p)}\left(\int_{0}^{1}\left[\theta^{s}\left|f^{\prime}(x)\right|^{q}+(1-\theta)^{s}\left|f^{\prime}\left(\lambda_{1}\right)\right|^{q}\right] d \theta\right)^{\frac{1}{q}} \\
& +\frac{\left(\lambda_{2}-x\right)^{\zeta}}{2} \sqrt[p]{\Xi_{\Phi, 2}^{\zeta}(x, p)}\left(\int_{0}^{1}\left[\theta^{s}\left|f^{\prime}(x)\right|^{q}+(1-\theta)^{s}\left|f^{\prime}\left(\lambda_{2}\right)\right|^{q}\right] d \theta\right)^{\frac{1}{q}} \\
= & \frac{\left(x-\lambda_{1}\right)^{\zeta}}{2} \sqrt[p]{\Xi_{\Phi, 1}^{\zeta}(x, p)} \sqrt[q]{\frac{\left.f^{\prime}(x)\right|^{q}+\left|f^{\prime}\left(\lambda_{1}\right)\right|^{q}}{s+1}} \\
& +\frac{\left(\lambda_{2}-x\right)^{\zeta}}{2} \sqrt[p]{\Xi_{\Phi, 2}^{\zeta}(x, p)} \sqrt[q]{\frac{\left|f^{\prime}(x)\right|^{q}+\left|f^{\prime}\left(\lambda_{2}\right)\right|^{q}}{s+1}} .
\end{aligned}
$$

The proof of Theorem 7 is completed.

We point out some particular cases of Theorem 7.

Corollary 11 Taking $x=\frac{\lambda_{1}+\lambda_{2}}{2}$ in Theorem 7, we get the following midpoint inequality via generalized conformable fractional integral operators:

$$
\begin{aligned}
\left|I_{f, \Sigma_{\Phi, 1}^{\zeta}, \Sigma_{\Phi, 2}^{\zeta}}\left(\frac{\lambda_{1}+\lambda_{2}}{2} ; \lambda_{1}, \lambda_{2}\right)\right| \\
\leq \frac{\left(\lambda_{2}-\lambda_{1}\right)^{\zeta}}{2^{\zeta+1} \sqrt[p]{s+1}} \sqrt[p]{\Xi_{\Phi}^{\zeta}(p)} \\
\quad \times\left\{\sqrt[q]{\left|f^{\prime}\left(\lambda_{1}\right)\right|^{q}+\left|f^{\prime}\left(\frac{\lambda_{1}+\lambda_{2}}{2}\right)\right|^{q}}+\sqrt[q]{\left|f^{\prime}\left(\frac{\lambda_{1}+\lambda_{2}}{2}\right)\right|^{q}+\left|f^{\prime}\left(\lambda_{2}\right)\right|^{q}}\right\}
\end{aligned}
$$

where

$$
\Xi_{\Phi}^{\zeta}(p)=\int_{0}^{1}\left(\int_{0}^{\theta} \frac{\Phi\left(u\left(\frac{\lambda_{2}-\lambda_{1}}{2}\right)\right)}{u}(1-u)^{\zeta-1} d u\right)^{p} d \theta
$$

Corollary 12 Taking $s=1$ in Theorem 7, we have the following inequality for convex function via generalized conformable fractional integral operators:

$$
\begin{aligned}
& \mid I_{f, \Sigma_{\Phi, 1}^{\zeta}, \Sigma_{\Phi, 2}^{\zeta}\left(x ; \lambda_{1}, \lambda_{2}\right) \mid} \\
& \leq \frac{\left(x-\lambda_{1}\right)^{\zeta}}{2} \sqrt[p]{\Xi_{\Phi, 1}^{\zeta}(x, p)} \sqrt[q]{\frac{\left|f^{\prime}(x)\right|^{q}+\left|f^{\prime}\left(\lambda_{1}\right)\right|^{q}}{2}} \\
& \quad+\frac{\left(\lambda_{2}-x\right)^{\zeta}}{2} \sqrt[p]{\Xi_{\Phi, 2}^{\zeta}(x, p)} \sqrt[q]{\frac{\left|f^{\prime}(x)\right|^{q}+\left|f^{\prime}\left(\lambda_{2}\right)\right|^{q}}{2}} .
\end{aligned}
$$


Corollary 13 Taking $\left|f^{\prime}\right| \leq K$ in Theorem 7 , we obtain

$$
\left|I_{f, \Sigma_{\Phi, 1}^{\zeta}, \Sigma_{\Phi, 2}^{\zeta}}\left(x ; \lambda_{1}, \lambda_{2}\right)\right| \leq \frac{K}{2} \sqrt[q]{\frac{2}{s+1}}\left\{\left(x-\lambda_{1}\right)^{\zeta} \sqrt[p]{\Xi_{\Phi, 1}^{\zeta}(x, p)}+\left(\lambda_{2}-x\right)^{\zeta} \sqrt[p]{\Xi_{\Phi, 2}^{\zeta}(x, p)}\right\}
$$

Theorem 8 Let $f:\left[\lambda_{1}, \lambda_{2}\right] \longrightarrow \mathbb{R}$ be a differentiable function on $\left(\lambda_{1}, \lambda_{2}\right)$. If $f^{\prime} \in L\left[\lambda_{1}, \lambda_{2}\right]$ and $\left|f^{\prime}\right|^{q}$ is s-convex in the second sense with $s \in(0,1]$, then for $q \geq 1$, we have following inequality for generalized conformable fractional integrals:

$$
\begin{aligned}
& \left|I_{f, \Sigma_{\Phi, 1}^{\zeta}, \Sigma_{\Phi, 2}^{\zeta}}\left(x ; \lambda_{1}, \lambda_{2}\right)\right| \\
& \leq \frac{\left(x-\lambda_{1}\right)^{\zeta}}{2}\left[M_{\Phi, 1}^{\zeta}(x)\right] \sqrt[1-\frac{1}{q}]{q} \sqrt{M_{\Phi, 2}^{s, \zeta}(x)\left|f^{\prime}(x)\right|^{q}+M_{\Phi, 3}^{s, \zeta}(x)\left|f^{\prime}\left(\lambda_{1}\right)\right|^{q}} \\
& +\frac{\left(\lambda_{2}-x\right)^{\zeta}}{2}\left[M_{\Phi, 4}^{\zeta}(x)\right] \sqrt[1-\frac{1}{q}]{q} \sqrt{M_{\Phi, 5}^{s, \zeta}(x)\left|f^{\prime}(x)\right|^{q}+M_{\Phi, 6}^{s, \zeta}(x)\left|f^{\prime}\left(\lambda_{2}\right)\right|^{q}},
\end{aligned}
$$

where

$$
\begin{aligned}
& M_{\Phi, 1}^{\zeta}(x)=\frac{\Psi_{\Phi}^{\zeta}}{\left(x-\lambda_{1}\right)^{\zeta-1}}-\frac{1}{\left(x-\lambda_{1}\right)^{\zeta}} \int_{\lambda_{1}}^{x} \Phi\left(\theta-\lambda_{1}\right)(x-\theta)^{\zeta-1} d \theta, \\
& M_{\Phi, 2}^{s, \zeta}(x)=\frac{\Psi_{\Phi}^{\zeta}}{(s+1)\left(x-\lambda_{1}\right)^{\zeta-1}}-\frac{1}{(s+1)\left(x-\lambda_{1}\right)^{\zeta+s}} \int_{\lambda_{1}}^{x} \Phi\left(\theta-\lambda_{1}\right)\left(\theta-\lambda_{1}\right)^{s}(x-\theta)^{\zeta-1} d \theta, \\
& M_{\Phi, 3}^{s, \zeta}(x)=\frac{1}{(s+1)\left(x-\lambda_{1}\right)^{\zeta+s}} \int_{\lambda_{1}}^{x} \frac{\Phi\left(\theta-\lambda_{1}\right)}{\theta-\lambda_{1}}(x-\theta)^{\zeta+s} d \theta, \\
& M_{\Phi, 4}^{\zeta}(x)=\frac{\Psi_{\Phi}^{\zeta}}{\left(\lambda_{2}-x\right)^{\zeta-1}}-\frac{1}{\left(\lambda_{2}-x\right)^{\zeta}} \int_{x}^{\lambda_{2}} \Phi\left(\lambda_{2}-\theta\right)(\theta-x)^{\zeta-1} d \theta, \\
& M_{\Phi, 5}^{s, \zeta}(x)=\frac{\Psi_{\Phi}^{\zeta}}{(s+1)\left(\lambda_{2}-x\right)^{\zeta-1}}-\frac{1}{(s+1)\left(\lambda_{2}-x\right)^{\zeta+s}} \int_{x}^{\lambda_{2}} \Phi(\theta-x)(\theta-x)^{s}\left(\lambda_{2}-\theta\right)^{\zeta-1} d \theta, \\
& M_{\Phi, 6}^{s, \zeta}(x)=\frac{1}{(s+1)\left(\lambda_{2}-x\right)^{\zeta+s}} \int_{x}^{\lambda_{2}} \frac{\Phi(\theta-x)}{\theta-x}\left(\lambda_{2}-\theta\right)^{\zeta+s} d \theta,
\end{aligned}
$$

and $\Psi_{\Phi}^{\zeta}$ is defined from (15).

Proof By Lemma 3.2, the $s$-convexity in the second sense of $\left|f^{\prime}\right|^{q}$, the well-known power mean inequality, and properties of the modulus we have

$$
\begin{aligned}
& \left|I_{f, \Sigma_{\Phi, 1}^{\zeta}, \Sigma_{\Phi, 2}^{\zeta}}\left(x ; \lambda_{1}, \lambda_{2}\right)\right| \\
& \leq \frac{\left(x-\lambda_{1}\right)^{\zeta}}{2} \int_{0}^{1} \Sigma_{\Phi, 1}^{\zeta}(x, \theta)\left|f^{\prime}\left(\theta x+(1-\theta) \lambda_{1}\right)\right| d \theta \\
& +\frac{\left(\lambda_{2}-x\right)^{\zeta}}{2} \int_{0}^{1} \Sigma_{\Phi, 2}^{\zeta}(x, \theta)\left|f^{\prime}\left(\theta x+(1-\theta) \lambda_{2}\right)\right| d \theta \\
& \leq \frac{\left(x-\lambda_{1}\right)^{\zeta}}{2}\left(\int_{0}^{1} \Sigma_{\Phi, 1}^{\zeta}(x, \theta) d \theta\right)^{1-\frac{1}{q}}\left(\int_{0}^{1} \Sigma_{\Phi, 1}^{\zeta}(x, \theta)\left|f^{\prime}\left(\theta x+(1-\theta) \lambda_{1}\right)\right|^{q} d \theta\right)^{\frac{1}{q}} \\
& +\frac{\left(\lambda_{2}-x\right)^{\zeta}}{2}\left(\int_{0}^{1} \Sigma_{\Phi, 2}^{\zeta}(x, \theta) d \theta\right)^{1-\frac{1}{q}}\left(\int_{0}^{1} \Sigma_{\Phi, 2}^{\zeta}(x, \theta)\left|f^{\prime}\left(\theta x+(1-\theta) \lambda_{2}\right)\right|^{q} d \theta\right)^{\frac{1}{q}}
\end{aligned}
$$




$$
\begin{aligned}
\leq & \frac{\left(x-\lambda_{1}\right)^{\zeta}}{2}\left[M_{\Phi, 1}^{\zeta}(x)\right]^{1-\frac{1}{q}}\left(\int_{0}^{1} \Sigma_{\Phi, 1}^{\zeta}(x, \theta)\left[\theta^{s}\left|f^{\prime}(x)\right|^{q}+(1-\theta)^{s}\left|f^{\prime}\left(\lambda_{1}\right)\right|^{q}\right] d \theta\right)^{\frac{1}{q}} \\
& +\frac{\left(\lambda_{2}-x\right)^{\zeta}}{2}\left[M_{\Phi, 4}^{\zeta}(x)\right]^{1-\frac{1}{q}}\left(\int_{0}^{1} \Sigma_{\Phi, 2}^{\zeta}(x, \theta)\left[\theta^{s}\left|f^{\prime}(x)\right|^{q}+(1-\theta)^{s}\left|f^{\prime}\left(\lambda_{2}\right)\right|^{q}\right] d \theta\right)^{\frac{1}{q}} \\
= & \frac{\left(x-\lambda_{1}\right)^{\zeta}}{2}\left[M_{\Phi, 1}^{\zeta}(x)\right] \sqrt[1-\frac{1}{q}]{q} \sqrt{M_{\Phi, 2}^{s, \zeta}(x)\left|f^{\prime}(x)\right|^{q}+M_{\Phi, 3}^{s, \zeta}(x)\left|f^{\prime}\left(\lambda_{1}\right)\right|^{q}} \\
& +\frac{\left(\lambda_{2}-x\right)^{\zeta}}{2}\left[M_{\Phi, 4}^{\zeta}(x)\right] \sqrt[1-\frac{1}{q}]{q} \sqrt{M_{\Phi, 5}^{s, \zeta}(x)\left|f^{\prime}(x)\right|^{q}+M_{\Phi, 6}^{s, \zeta}(x)\left|f^{\prime}\left(\lambda_{2}\right)\right|^{q}}
\end{aligned}
$$

The proof of Theorem 8 is completed.

We point out some particular cases of Theorem 8 .

Corollary 14 Taking $x=\frac{\lambda_{1}+\lambda_{2}}{2}$ in Theorem 8, we get the following midpoint inequality via generalized conformable fractional integral operators:

$$
\begin{aligned}
\mid I_{f, \Sigma_{\Phi, 1}^{\zeta}}, \Sigma_{\Phi, 2}^{\zeta} & \left(\frac{\lambda_{1}+\lambda_{2}}{2} ; \lambda_{1}, \lambda_{2}\right) \mid \\
\leq & \frac{\left(\lambda_{2}-\lambda_{1}\right)^{\zeta}}{2^{\zeta+1}} \\
& \times\left\{\left[M_{\Phi, 1}^{\zeta}\left(\frac{\lambda_{1}+\lambda_{2}}{2}\right)\right]^{1-\frac{1}{q}}\right. \\
& \times \sqrt[q]{M_{\Phi, 2}^{s, \zeta}\left(\frac{\lambda_{1}+\lambda_{2}}{2}\right)\left|f^{\prime}\left(\frac{\lambda_{1}+\lambda_{2}}{2}\right)\right|^{q}+M_{\Phi, 3}^{s, \zeta}\left(\frac{\lambda_{1}+\lambda_{2}}{2}\right)\left|f^{\prime}\left(\lambda_{1}\right)\right|^{q}} \\
& +\left[M_{\Phi, 4}^{\zeta}\left(\frac{\lambda_{1}+\lambda_{2}}{2}\right)\right]^{1-\frac{1}{q}} \\
& \left.\times \sqrt[q]{M_{\Phi, 5}^{s, \zeta}\left(\frac{\lambda_{1}+\lambda_{2}}{2}\right)\left|f^{\prime}\left(\frac{\lambda_{1}+\lambda_{2}}{2}\right)\right|^{q}+M_{\Phi, 6}^{s, \zeta}\left(\frac{\lambda_{1}+\lambda_{2}}{2}\right)\left|f^{\prime}\left(\lambda_{2}\right)\right|^{q}}\right\} .
\end{aligned}
$$

Corollary 15 Taking $s=1$ in Theorem 8, we have the following inequality for convex functions via generalized conformable fractional integral operators:

$$
\begin{aligned}
& \left|I_{f, \Sigma_{\Phi, 1}^{\zeta}, \Sigma_{\Phi, 2}^{\zeta}}\left(x ; \lambda_{1}, \lambda_{2}\right)\right| \\
& \leq \frac{\left(x-\lambda_{1}\right)^{\zeta}}{2}\left[M_{\Phi, 1}^{\zeta}(x)\right] \sqrt[1-\frac{1}{q}]{q} \sqrt{M_{\Phi, 2}^{\zeta}(x)\left|f^{\prime}(x)\right|^{q}+M_{\Phi, 3}^{\zeta}(x)\left|f^{\prime}\left(\lambda_{1}\right)\right|^{q}} \\
& +\frac{\left(\lambda_{2}-x\right)^{\zeta}}{2}\left[M_{\Phi, 4}^{\zeta}(x)\right] \sqrt[1-\frac{1}{q}]{q} M_{\Phi, 5}^{\zeta}(x)\left|f^{\prime}(x)\right|^{q}+M_{\Phi, 6}^{\zeta}(x)\left|f^{\prime}\left(\lambda_{2}\right)\right|^{q},
\end{aligned}
$$

where

$$
\begin{aligned}
& M_{\Phi, 2}^{\zeta}(x)=\frac{\Psi_{\Phi}^{\zeta}}{2\left(x-\lambda_{1}\right)^{\zeta-1}}-\frac{1}{2\left(x-\lambda_{1}\right)^{\zeta+1}} \int_{\lambda_{1}}^{x} \Phi\left(\theta-\lambda_{1}\right)\left(\theta-\lambda_{1}\right)(x-\theta)^{\zeta-1} d \theta, \\
& M_{\Phi, 3}^{\zeta}(x)=\frac{1}{2\left(x-\lambda_{1}\right)^{\zeta+1}} \int_{\lambda_{1}}^{x} \frac{\Phi\left(\theta-\lambda_{1}\right)}{\theta-\lambda_{1}}(x-\theta)^{\zeta+1} d \theta
\end{aligned}
$$




$$
M_{\Phi, 5}^{\zeta}(x)=\frac{\Psi_{\Phi}^{\zeta}}{2\left(\lambda_{2}-x\right)^{\zeta-1}}-\frac{1}{2\left(\lambda_{2}-x\right)^{\zeta+1}} \int_{x}^{\lambda_{2}} \Phi(\theta-x)(\theta-x)\left(\lambda_{2}-\theta\right)^{\zeta-1} d \theta
$$

and

$$
M_{\Phi, 6}^{\zeta}(x)=\frac{1}{2\left(\lambda_{2}-x\right)^{\zeta+1}} \int_{x}^{\lambda_{2}} \frac{\Phi(\theta-x)}{\theta-x}\left(\lambda_{2}-\theta\right)^{\zeta+1} d \theta .
$$

Corollary 16 Taking $\left|f^{\prime}\right| \leq K$ in Theorem 8 , we obtain

$$
\begin{aligned}
&\left|I_{f, \Sigma_{\Phi, 1}^{\zeta}, \Sigma_{\Phi, 2}^{\zeta}}\left(x ; \lambda_{1}, \lambda_{2}\right)\right| \\
& \leq \frac{K}{2}\left\{\left(x-\lambda_{1}\right)^{\zeta}\left[M_{\Phi, 1}^{\zeta}(x)\right] \sqrt[1-\frac{1}{q}]{q} \sqrt{M_{\Phi, 2}^{s, \zeta}(x)+M_{\Phi, 3}^{s, \zeta}(x)}\right. \\
&\left.+\left(\lambda_{2}-x\right)^{\zeta}\left[M_{\Phi, 4}^{\zeta}(x)\right] \sqrt[1-\frac{1}{q}]{q} \sqrt{M_{\Phi, 5}^{s, \zeta}(x)+M_{\Phi, 6}^{s, \zeta}(x)}\right\} .
\end{aligned}
$$

Remark 4. Applying our Theorems 5, 6, 7, and 8 to suitable functions $\Phi(\theta)=\theta, \frac{\theta^{\xi}}{\Gamma(\xi)}, \frac{\theta^{\frac{\xi}{k}}}{k \Gamma_{k}(\xi)}$, $\Phi(\theta)=\theta\left(\lambda_{2}-\theta\right)^{\xi-1}$ for $\xi \in(0,1), \Phi(\theta)=\frac{\theta}{\xi} \exp (-A \theta)$, where $A=\frac{1-\xi}{\xi}$ for $\xi \in(0,1)$, and $f$ such that $\left|f^{\prime}\right|^{q}$ is an $s$-convex function $\left(f(x)=x^{2}\right.$, etc.), we can construct some new generalized conformable fractional integral inequalities. Also, we can obtain several new general fractional integral inequalities using special means (arithmetic, geometric, logarithmic, etc.). Finally, some new bounds for the midpoint and trapezium quadrature formula using our results can be provided as well. We omit their proofs, and the details are left to the interested readers.

\section{Conclusion}

Trapezium-type integral inequalities for functions of divers natures are useful in numerical computations. Using the generalized conformable fractional integral operators defined in our paper, the interested reader can obtain in a similar way new results for different operators, such as $k$-Riemann-Liouville fractional integral, Katugampola fractional integrals, the conformable fractional integral, $(p, q)$-quantum calculus, Hadamard fractional integrals, and so on. These results can be applied in convex analysis, optimization, probability, and also different areas of pure and applied sciences. The ideas and techniques of this paper may stimulate further research in the fascinating field of integral inequalities.

Acknowledgements

We thank the reviewers for their great comments to improve the quality of our paper.

Funding

This work was supported by National Natural Science Foundation of China (11761083)

Availability of data and materials

Not applicable.

Competing interests

The authors declare no conflict of interest.

Authors' contributions

The authors have worked equally when writing this paper. All authors read and approved the final manuscript.

\section{Author details}

${ }^{1}$ Department of Mathematics, Faculty of Technical Science, University Ismail Qemali, Vlora, Albania. ${ }^{2}$ Department of Mathematics, University of Management and Technology, Sialkot, Pakistan. ${ }^{3}$ School of Information Science and Technology, Yunnan Normal University, Kunming, China. ${ }^{4}$ Department of Mathematics, University of Sargodha, Sargodha, Pakistan. 


\section{Publisher's Note}

Springer Nature remains neutral with regard to jurisdictional claims in published maps and institutional affiliations.

\section{Received: 25 November 2019 Accepted: 28 April 2020 Published online: 14 May 2020}

\section{References}

1. Abdeljawad, T.: On conformable fractional calculus. J. Comput. Appl. Math. 279, 57-66 (2015)

2. Aslani, S.M., Delavar, M.R., Vaezpour, S.M.: Inequalities of Fejér type related to generalized convex functions with applications. Int. J. Anal. Appl. 16(1), 38-49 (2018)

3. Chen, F.X., Wu, S.H.: Several complementary inequalities to inequalities of Hermite-Hadamard type for s-convex functions. J. Nonlinear Sci. Appl. 9(2), 705-716 (2016)

4. Chu, Y.M., Khan, M.A., Khan, T.U., Ali, T.: Generalizations of Hermite-Hadamard type inequalities for MT-convex functions. J. Nonlinear Sci. Appl. 9(5), 4305-4316 (2016)

5. Delavar, M.R., De La Sen, M.: Some generalizations of Hermite-Hadamard type inequalities. SpringerPlus 5, 166 (2016)

6. Delavar, M.R., Dragomir, S.S.: On $\eta$-convexity. Math. Inequal. Appl. 20, 203-216 (2017)

7. Dragomir, S.S., Agarwal, R.P.: Two inequalities for differentiable mappings and applications to special means of real numbers and trapezoidal formula. Appl. Math. Lett. 11(5), 91-95 (1998)

8. El-Ajou, A., Oqielat, M.N., Al-Zhour, Z., Kumar, S., Momani, S.: Solitary solutions for time-fractional nonlinear dispersive PDEs in the sense of conformable fractional derivative. Chaos 29, 093102 (2019)

9. Farid, G., Rehman, A.U.: Generalizations of some integral inequalities for fractional integrals. Ann. Math. Sil. 31, 14 (2017)

10. Goufo, E.F.D., Kumar, S., Mugisha, S.B.: Similarities in a fifth-order evolution equation with and with no singular kernel. Chaos Solitons Fractals 130, 109467 (2020)

11. Hristov, J.: Response functions in linear viscoelastic constitutive equations and related fractional operators. Math. Model. Nat. Phenom. 14(3), 1-34 (2019)

12. Huang, C.J., Rahman, G., Nisar, K.S., Ghaffar, A., Qi, F.: Some inequalities of the Hermite-Hadamard type for k-fractional conformable integrals. Aust. J. Math. Anal. Appl. 16(1), 7 (2019)

13. Hudzik, H., Maligranda, L.: Some remarks on s-convex functions. Aequ. Math. 48, 100-111 (1994)

14. Jleli, M., Samet, B.: On Hermite-Hadamard type inequalities via fractional integral of a function with respect to another function. J. Nonlinear Sci. Appl. 9, 1252-1260 (2016)

15. Kashuri, A., Liko, R.: Some new Hermite-Hadamard type inequalities and their applications. Studia Sci. Math. Hung. 56(1), 103-142 (2019)

16. Katugampola, U.N.: New approach to a generalized fractional integral. Appl. Math. Comput. 218(3), 860-865 (2011)

17. Khalil, R., Horani, M.A., Yousef, A., Sababheh, M.: A new definition of fractional derivatives. J. Comput. Appl. Math. 264, 65-70 (2014)

18. Khan, M.A., Ali, T., Dragomir, S.S., Sarikaya, M.Z.: Hermite-Hadamard type inequalities for conformable fractional integrals. Revista de la Real Academia de Ciencias Exactas, Físicas y Naturales. Serie A. Matemáticas (2017)

19. Khan, M.A., Chu, Y.M., Kashuri, A., Liko, R., Ali, G.: Conformable fractional integrals versions of Hermite-Hadamard inequalities and their generalizations. J. Funct. Spaces 2018, Article ID 6928130 (2018)

20. Kumar, S., Kumar, A., Momani, S., Aldhaifallah, M., Nisar, K.S.: Numerical solutions of nonlinear fractional model arising in the appearance of the strip patterns in two-dimensional systems. Adv. Differ. Equ. 2019, 413 (2019)

21. Liu, W., Wen, W., Park, J.: Hermite-Hadamard type inequalities for MT-convex functions via classical integrals and fractional integrals. J. Nonlinear Sci. Appl. 9, 766-777 (2016)

22. Mihai, M.V.: Some Hermite-Hadamard type inequalities via Riemann-Liouville fractional calculus. Tamkang J. Math. 44(4), 411-416 (2013)

23. Odibat, Z., Kumar, S.: A robust computational algorithm of homotopy asymptotic method for solving systems of fractional differential equation. J. Comput. Nonlinear Dyn. 14(8), 081004 (2019)

24. Omotoyinbo, O., Mogbodemu, A.: Some new Hermite-Hadamard integral inequalities for convex functions. Int. J. Sci. Innov. Technol. 1(1), 1-12 (2014)

25. Özdemir, M.E., Dragomir, S.S., Yildiz, C.: The Hadamard's inequality for convex function via fractional integrals. Acta Math. Sci. 33(5), 153-164 (2013)

26. Qi, F., Mohammed, P.O., Yao, J., Yao, Y.: Generalized fractional integral inequalities of Hermite-Hadamard type for $(\alpha, m)$-convex functions. J. Inequal. Appl. 2019, 135 (2019)

27. Qi, F., Xi, B.Y.: Some integral inequalities of Simpson type for GA- $\epsilon$-convex functions. Georgian Math. J. 20(5), 775-788 (2013)

28. Sarikaya, M.Z., Ertuğral, F.: On the generalized Hermite-Hadamard inequalities (2017) Submitted. https://www.researchgate.net/publication/321760443

29. Sarikaya, M.Z., Yildirim, H.: On generalization of the Riesz potential. Indian J. Math. Sci. 3(2), 231-235 (2007)

30. Set, E., Gözpinar, A.: A study On Hermite-Hadamard type inequalities for $s$-convex functions via conformable fractional integrals (2016). https://www.researchgate.net/publication/303382148

31. Set, E., Noor, M.A., Awan, M.U., Gözpinar, A.: Generalized Hermite-Hadamard type inequalities involving fractional integral operators. J. Inequal. Appl. 2017, 169 (2017)

32. Set, E., Sarikaya, M.Z., Gözpinar, A.: Some Hermite-Hadamard type inequalities for convex functions via conformable fractional integrals and related inequalities. Creative Math. Inform. 26(2), 221-229 (2017)

33. Sharma, B., Kumar, S., Cattani, C., Baleanu, D.: Nonlinear dynamics of Cattaneo-Christov heat flux model for third-grade power-law fluid. J. Comput. Nonlinear Dyn. 15(1), 011009 (2020)

34. Wang, H., Du, T.S., Zhang, Y.: $k$-fractional integral trapezium-like inequalities through $(h, m)$-convex and $(\xi, m)$-convex mappings. J. Inequal. Appl. 2017(311), 20 (2017)

35. Xi, B.Y., Qi, F: Some integral inequalities of Hermite-Hadamard type for convex functions with applications to means. J. Funct. Spaces Appl. 2012, Article ID 980438 (2012)

36. Zhang, X.M., Chu, Y.M., Zhang, X.H.: The Hermite-Hadamard type inequality of GA-convex functions and its applications. J. Inequal. Appl. 2010, Article ID 507560 (2010) 
37. Zhang, Y., Du, T.S., Wang, H., Shen, Y.J., Kashuri, A.: Extensions of different type parameterized inequalities for generalized $(m, h)$-preinvex mappings via $k$-fractional integrals. J. Inequal. Appl. 2018(49), 30 (2018)

Submit your manuscript to a SpringerOpen ${ }^{\odot}$ journal and benefit from:

- Convenient online submission

- Rigorous peer review

- Open access: articles freely available online

- High visibility within the field

- Retaining the copyright to your article

Submit your next manuscript at $\gg$ springeropen.com 